\title{
Private Information
}

Retrieval from Coded

\section{Storage}

Razane Tajeddine

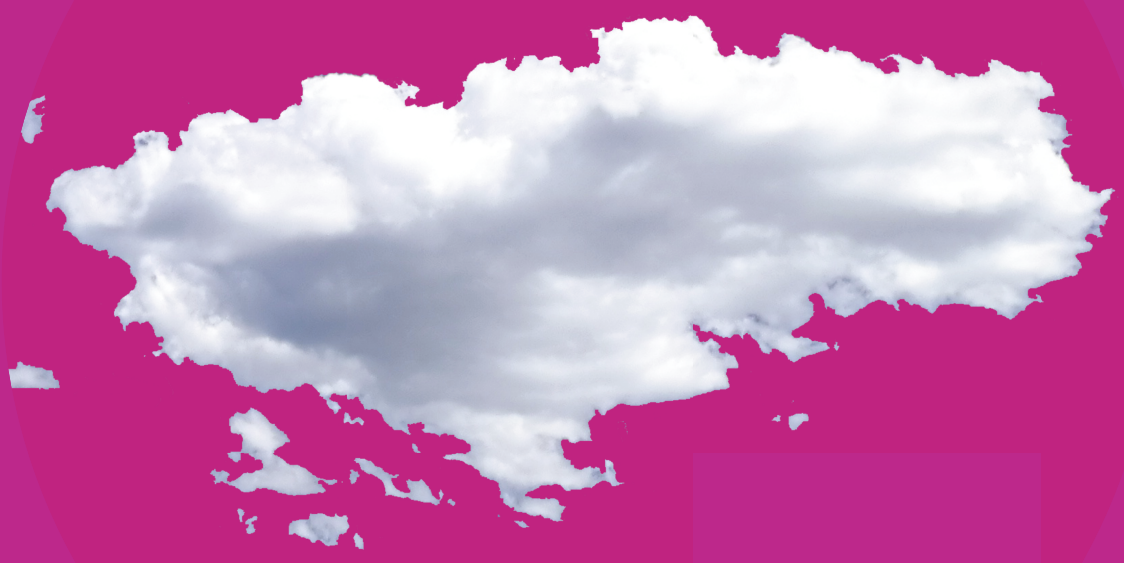




\section{Private Information Retrieval from Coded Storage}

Razane Tajeddine

A doctoral dissertation completed for the degree of Doctor of Science (Technology) to be defended, with the permission of the Aalto University School of Science, at a public examination held at the lecture hall M1 of the school on 07 March 2019 at 14:00. 
Supervising professors

Prof. Camilla Hollanti

Aalto University

Finland

\section{Thesis advisors}

Prof. Camilla Hollanti

Aalto University

Finland

Prof. Salim El Rouayheb

Rutgers University

United States

Dr. Oliver Gnilke

Aalto University

Finland

Preliminary examiners

Prof. Mikael Skoglund

KTH

Sweden

Prof. Sennur Ulukus

University of Maryland

United States

\section{Opponents}

Prof. Simon Blackburn

Royal Holloway, University of London

United Kingdom

Aalto University publication series

DOCTORAL DISSERTATIONS 29/2019

(c) 2019 Razane Tajeddine

ISBN 978-952-60-8421-3 (printed)

ISBN 978-952-60-8422-0 (pdf)

ISSN 1799-4934 (printed)

ISSN 1799-4942 (pdf)

http://urn.fi/URN:ISBN:978-952-60-8422-0

Unigrafia Oy

Helsinki 2019

Finland 
Author

Razane Tajeddine

Name of the doctoral dissertation

Private Information Retrieval from Coded Storage

\begin{tabular}{|c|c|}
\hline Unit Department of Mathematics and Systems Analysis & \\
\hline Series Aalto University publication series DOCTORAL DIS & SSERTATIONS 29/2019 \\
\hline \multicolumn{2}{|l|}{ Field of research Mathematics } \\
\hline Manuscript submitted 10 December 2018 & Date of the defence 7 March 2019 \\
\hline Permission to publish granted (date) 30 January 2019 & Language English \\
\hline$\bigotimes$ Article dissertation & $\square$ Essay dissertation \\
\hline
\end{tabular}

\section{Abstract}

Along with the technological advancements and the remarkable growth of digital data storage, new challenges arise regarding the reliabilty of the digital data storage and the privacy of the users. A distributed storage system (DSS) can store big amounts of data for a long time over possibly unreliable servers. As for the privacy concern of the users, much effort has been spent in trying to find systems and schemes that allow users to communicate, search, upload and download files while maintaining their privacy. One of those schemes is private information retrieval (PIR).

PIR allows the users to hide the identity of files they request from a DSS. In other words, a PIR scheme allows the user to retrieve any file from a DSS without revealing the file identity to any of the servers. It goes without saying that, to achieve privacy, the user should download a bigger amount of data than the size of the desired file. The extra amount of data downloaded is quantified by the communication complexity of a PIR scheme. Naturally, downloading all the data from the DSS hides the identity of the desired file, but has a very high communication complexity, especially as the number of files in a DSS is typically very large. Moreover, this also raises the question of the server privacy, meaning that the user should not be able to retrieve any extra information about the undesired files while retrieving a certain file. Schemes taking into account the server privacy are called symmetric PIR (SPIR) schemes.

This thesis focuses on constructing PIR schemes for different DSSs with low communication cost. We study different DSSs which reduce the storage cost of the system while providing reliability. We construct PIR schemes on such systems, where we consider both cases, the case where the servers in the DSS do not communicate, and the case where some subsets of the servers communicate in an effort to figure out the desired file identity. Moreover, we consider the SPIR problem, and consider the case where some servers may be unresponsive, such that they do not respond to the sent query, and the case where the servers may be malicious or unsynchronized and thus give false information to the user. Last but not least, this thesis also considers schemes providing privacy for users requesting data from a DSS over a random linear network.

Keywords Privacy, Information Retrieval, Coding, Storage Systems, Reed-Solomon Codes, Regenrating Codes, Networks

\begin{tabular}{lc}
\hline ISBN (printed) $978-952-60-8421-3$ & ISBN (pdf) $978-952-60-8422-0$ \\
\hline ISSN (printed) $1799-4934$ & ISSN (pdf) $1799-4942$ \\
\hline Location of publisher Helsinki & Location of printing Helsinki Year 2019 \\
\hline Pages 144 & urn http://urn.fi/URN:ISBN:978-952-60-8422-0 \\
\hline
\end{tabular}





\section{Preface}

The research work for this doctoral thesis has been carried out at the Department of Mathematics and Systems Analysis, at Aalto University.

Foremost, I want to thank my supervisor Prof. Camilla Hollanti for her continuous support, motivation, and inspiration. I want to also thank my thesis advisors Prof. Oliver Gnilke and Prof. Salim El Rouayheb for their expertise and guidance. My gratitude also goes toward Prof. Antonia Wachter-Zeh, Prof. David Karpuk, and Dr. Ragnar Freij-Hollanti, working with them was always a pleasure.

I also would like to thank all the people who have been part of our research group at Aalto at any point in time for making this a great place to be.

I want to thank Prof. Sennur Ulukus and Prof. Mikael Skoglund for acting as external examiners of this dissertation, and Prof. Simon Blackburn for agreeing to act as my opponent.

I am very grateful for my friends, who were always providing me with unfailing support and continuous encouragement throughout my years of study and through the process of researching and writing this thesis.

Last but not least, I would like to express my deepest gratitude to my family, without whom none of this would have been possible. The moral support I got from you is incomparable. Thank you.

Helsinki, February 2, 2019,

Razane Tajeddine 
Preface 


\section{Contents}

$\begin{array}{ll}\text { Preface } & 1\end{array}$

$\begin{array}{ll}\text { Contents } & 3\end{array}$

$\begin{array}{ll}\text { List of Publications } & 5\end{array}$

$\begin{array}{ll}\text { Author's Contribution } & 7\end{array}$

$\begin{array}{lr}\text { 1. Introduction } & 9\end{array}$

2. Coding Theory and Applications: an Overview 13

2.1 Preliminary Definitions . . . . . . . . . . . . . . 13

2.2 Maximum Distance Separable (MDS) Codes . . . . . . . . . 15

2.2.1 Reed-Solomon (RS) codes . . . . . . . . . . . 16

2.2.2 Reed-Solomon Codes for Storage . . . . . . . . . 16

2.2.3 Star Product of RS Codes . . . . . . . . . . . . 17

2.3 Regenerating Codes . . . . . . . . . . . . . . . . . . 19

2.3.1 Product-Matrix (PM) Constructions . . . . . . . . 19

2.3.2 PM Codes in the MBR Setting [33] . . . . . . . . 20

2.3.3 PM Codes in the MSR Setting . . . . . . . . . . . . 21

2.4 Network Coding . . . . . . . . . . . . . . . . . . 21

2.5 Maximum Rank Distance (MRD) Codes . . . . . . . . . . . 22

2.5.1 Star Product of Gabidulin Codes . . . . . . . . . . 23

3. Private Information Retrieval (PIR) 25

3.1 Definition . . . . . . . . . . . . . . 25

3.2 Parameters of a PIR Scheme . . . . . . . . . . . . 26

3.3 PIR Schemes on Maximum Distance Separable (MDS) Codes 28

3.4 Regenerating Codes and PIR . . . . . . . . . . . 34 
3.4.1 Minimum Bandwidth Regenerating (MBR) Code Con-

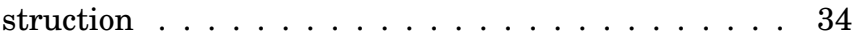

3.4.2 Minimum Storage Regenerating (MSR) Code Construction ...................... 38

3.5 PIR with Byzantine Servers . . . . . . . . . . . . . . . 39

3.5.1 Symmetric PIR scheme . . . . . . . . . . . . . . . 44

3.6 Network Coding with PIR . . . . . . . . . . . . . . 44

3.6.1 Error-Free Network . . . . . . . . . . . . . . . 46

3.6.2 Network with Errors . . . . . . . . . . . . . . . . 49

4. Conclusions $\quad 51$

$\begin{array}{ll}\text { Bibliography } & 53\end{array}$

$\begin{array}{ll}\text { Publications } & 57\end{array}$ 


\section{List of Publications}

This thesis consists of an overview and of the following publications which are referred to in the text by their Roman numerals.

I Razane Tajeddine, Oliver W. Gnilke, and Salim El Rouayheb. Private information retrieval from MDS coded data in distributed storage systems. IEEE Transactions on Information Theory, Volume 64, Issue 11, pp. 7081 - 7093, Nov 2018.

II Razane Tajeddine and Salim El Rouayheb. Robust Private Information Retrieval on Coded Data. In IEEE International Symposium on Information Theory (ISIT), 5 pages, 2017.

III Razane Tajeddine, Oliver W. Gnilke, David Karpuk, Ragnar FreijHollanti, Camilla Hollanti, and Salim El Rouayheb. Private Information Retrieval Schemes for Coded Data with Arbitrary Collusion Patterns. In IEEE International Symposium on Information Theory (ISIT), 5 pages, 2017.

IV Razane Tajeddine, Oliver W. Gnilke, David Karpuk, Ragnar FreijHollanti, and Camilla Hollanti. Private Information Retrieval from Coded Storage Systems with Colluding, Byzantine, and Unresponsive Servers. Accepted in IEEE Transactions on Information Theory, 9 pages, 2018.

V Julien Lavauzelle, Razane Tajeddine, Ragnar Freij-Hollanti, and Camilla Hollanti. Private Information Retrieval Schemes with Regenerating Codes. Submitted to IEEE Transactions on Information Theory, 26 pages, 2018.

VI Razane Tajeddine, Antonia Wachter-Zeh, and Camilla Hollanti. Private Information Retrieval over Networks. Submitted to IEEE Trans- 
actions on Information Forensics and Security, 10 pages, 2018. 


\section{Author's Contribution}

Publication I: "Private information retrieval from MDS coded data in distributed storage systems"

The author largely wrote the article and derived the scheme constructions.

Publication II: "Robust Private Information Retrieval on Coded Data"

The author largely wrote the article and derived the technical results.

Publication III: "Private Information Retrieval Schemes for Coded Data with Arbitrary Collusion Patterns"

The author largely wrote the article and contributed to deriving the technical details.

Publication IV: "Private Information Retrieval from Coded Storage Systems with Colluding, Byzantine, and Unresponsive Servers"

The author largely wrote the article and contributed in deriving the technical results.

Publication V: "Private Information Retrieval Schemes with Regenerating Codes"

The first two authors of the article contributed equally to the article, 
largely wrote the article and established the technical results.

\section{Publication VI: "Private Information Retrieval over Networks"}

The author largely wrote the article and derived the technical results. 


\section{Introduction}

With the current increase in digital data, there is a need for reliable and flexible storage systems. Storage nodes, e.g., disks or servers, can be unreliable when it comes to storing data for long periods of time. In case of a node failure, if there is no backup, user data could get lost with no possibility of recovering it. A distributed storage system (DSS) is a system consisting of several servers, which act together as a storage system. It is used to store data reliably such that, if a small subset of servers fails, the user can recover the data from other servers. For this sake, the storage system has to contain redundancy. It is then straightforward to see that one way to store information reliably and redundantly is to simply replicate the data on multiple servers. For storage efficiency, however, it is better to use erasure coding techniques [14], e.g., maximum distance seperable (MDS) codes, regenerating codes, or maximum rank distance (MRD) codes.

Nowadays, storage systems are used increasingly, be it for online storage or simple peer-to-peer networks. This raises the concern of user privacy. User privacy can be achieved by hiding the identity of the user, known as anonymity, or hiding the identity of the file desired by the user, known as private information retrieval (PIR). User anonymity is achieved among the set of users utilizing the storage system, i.e., among the potential users [32]. The onion router, or Tor in short, was built such that the user's anonymity is protected by connecting to the servers through virtual tunnels, making it near impossible for the sites to trace the user request back to their location [20,41]. However, anonymity is not always feasible, especially in cases where user authentication is desired, e.g., Netflix or other paid services.

On the other hand, PIR schemes give the user the ability to ask for a file, without revealing the identity of that file to any of the servers. One way 
of achieving privacy, in the PIR sense, is to download all the files stored in the system. This solution gives the user privacy, but it is very inefficient, due to the possibly very large size of the download. The problem of constructing PIR schemes was first introduced by Chor et al. in 1995 [10]. In this setting, an $m$-bit string, $\left\{x^{1}, \ldots, x^{m}\right\}$, is considered to be replicated on $n$ servers, and the user wants to retrieve bit $x^{i}$ from this DSS privately. The authors devise a PIR scheme that reduces the communication complexity, i.e., the number of the uploaded and downloaded bits, when the number of servers in the system is at least two. Moreover, it was also proven that when the data is stored on a single server, i.e., $n=1$, and the server has unlimited computational power, the user can only achieve privacy by downloading all $m$ bits.

However, in 1997, Kushilevitz and Ostrovsky [26] revisited the PIR problem, while assuming some limits on the computational power of the servers in the DSS. The authors then show that the communication complexity of the PIR scheme on a single server can be reduced by assuming the hardness of the quadratic residuoisity problem. Later in [8], the authors show that under the assumption that the servers cannot solve the $\phi$-hiding problem, the communication complexity of a single server PIR scheme can be further reduced. Despite that this setting allows the user to achieve privacy on a single server without downloading all the files, these schemes have high computational complexity on the server side. Moreover, in these schemes, privacy is not fully guaranteed, but relies on hardness assumptions which are not proven.

In subsequent years, significant work was done on constructing PIR schemes with low communication complexity on a system with data replicated on multiple servers, without any assumptions on their computational power [5, 6, 16, 45]. In more recent works, the PIR setting assumes a DSS storing $m$ files $\left\{x^{1}, \ldots, x^{m}\right\}$, where the file size is much larger than the number of files. In this setting, the request (query) size is generally independent of the file size, and thus, the number of communicated bits can be estimated by the number of downloaded bits only. The PIR rate is then measured as the ratio of the size of the desired file to the total size of the downloaded data. The PIR capacity is the highest achievable PIR rate in a certain setting. In 2017, Sun and Jafar [38] found the capacity of PIR on a replicated system with $n$ servers and $m$ files.

In 2014, Shah et al. [36] initiated a line of work where the data is assumed to be encoded on the DSS using an erasure code. In this work, 
the authors show that only one extra bit of download is enough to achieve PIR, assuming that the number of servers is exponential in the number of files. Later in [9], the authors derive bounds on the tradeoff between storage cost and download cost for linearly coded data. The PIR capacity for an $[n, k]$ MDS coded system was found in [4], and in Publication I we construct a PIR scheme achieving the asymptotic capacity, i.e., the capacity of the PIR scheme when the number of files, $m$, is unbounded. PIR schemes on arbitrary linear storage codes were constructed in [25].

In all the previously mentioned works, the servers are assumed to be non-communicating. In general, however, the servers can be assumed to communicate or collude in an effort to deduce the identity of the desired file. The capacity for PIR from a replicated DSS with colluding servers was found in [39]. For a DSS with coded databases, the capacity of PIR with colluding servers is still an open question. However, a PIR scheme on a DSS encoded using an $[n, k]$ Reed-Solomon (RS) code was devised in [18], and the PIR capacity was conjectured. A counterexample was then found which settled that the conjecture was false for a coded storage system with two files, $m=2$. For an unbounded number of files, however, the conjectured asymptotic capacity in [18] is still unsettled.

In addition to the above settings, much work has been done in the case where some servers are unresponsive, and some send incorrect data. The servers may send incorrect data for one of two reasons, either they are byzantine (malicious), or they are unsynchronized, i.e., have outdated information. The problem of constructing PIR schemes on replicated data with byzantine servers was considered in $[2,7,13]$. The capacity of PIR on a DSS, where the data is replicated on multiple servers with some colluding servers and some byzantine servers was found in [3]. In Publication II, an adaptive PIR scheme was constructed on an $[n, k]$ MDS coded database which achieves the asymptotic PIR capacity. Furthermore, in Publication IV, we construct a PIR scheme on a DSS encoded using an RS code, allowing for colluding, unresponsive, and byzantine servers. Another similar setting was introduced in [17], where the servers are assumed to be unsynchronized. In this setting, the authors construct an adaptive PIR scheme.

In more recent years, the notion of symmetric PIR (SPIR) was studied. In the symmetric PIR setting, the user wants to hide the identity of the desired file, and the servers want to share information only about the file desired by the user. In other words, the privacy of both ends of the 
communication is considered. A first SPIR scheme on replicated data with non-colluding servers was constructed in [40]. The authors also found the capacity of SPIR on replicated data. Later, SPIR schemes were devised on MDS coded data with non-colluding servers in [44]. The authors later quantified the capacity of linear SPIR schemes on MDS coded data with colluding servers in [42], and the capacity of SPIR schemes on replicated data with colluding, byzantine, and unresponsive servers in [43].

The work in this thesis focuses on PIR schemes on coded data. The rest of the thesis is organized as follows. In Chapter 2, we give some basics of coding theory, we define formally a distributed storage system, and we recall some erasure codes and their properties. The results of the publications in this thesis are discussed in Chapter 3. In Section 3.1, we discuss more formally the definition of PIR and the basic schemes introduced in [10]. Then, in Section 3.2, we define more formally the settings of a PIR scheme used in this work. In Sections 3.3 to 3.6, we discuss our results on PIR schemes on certain erasure codes. Finally, Chapter 4 provides concluding remarks and future work directions. 


\section{Coding Theory and Applications: an Overview}

Data storage is a major concern nowadays due to the massive increase in digital data. The concept of distributed storage is to store data reliably on a decentralized network of servers. As servers may be unreliable, distributed storage systems (DSSs) introduce redundancy to the system. Redundancy can be introduced by replicating the data on the servers, which allows for recovery of a failed server, or reconstruction of a file by contacting any other server. In terms of coding, this is referred to as a repetition code. Erasure codes are an evolution of repetition codes offering a lower storage overhead. In this setting, the files are divided into $k$ parts which are then encoded into $n$ symbols. Good erasure codes are built such that any $k$ out of the $n$ symbols are sufficient to recover the files. In this chapter, we give the basic properties of some erasure codes.

\subsection{Preliminary Definitions}

Let $q$ be a power of a prime. We denote the field of $q$ elements by $\mathbb{F}_{q}$. For any two vectors $u, v \in \mathbb{F}_{q}^{n}$, we denote their inner product by $\langle u, v\rangle$. We also denote by $[n]$ the set $\{1,2, \ldots, n\}$. An identity matrix $I_{k \times k}$ is a square $k \times k$ matrix with ones on the main diagonal and zeros elsewhere.

To store messages reliably, they are encoded into codewords. An encoding is an injective mapping from a vector space of dimension $k$ into a vector space of dimension $n$, where $n \geq k$ [34]. These codewords form a code. A $q$-ary code of length $n$ is a nonempty subset of $\mathbb{F}_{q}^{n}$.

A systematic code is a code such that the output data contains the uncoded input data. On the other hand, in a non-systematic code, the input symbols are contained in the output as coded symbols only.

Linear codes are a subclass of codes such that any linear combination of two codewords of $\mathcal{C}$ is also a codeword of $\mathcal{C}$. In this work, we only consider 
linear codes, thus we briefly use code to refer to a linear code. A code $\mathcal{C}$ has three important parameters: the dimension $k$, length $n$, and minimum distance $d_{\min }$. We call such a code an $\left[n, k, d_{\min }\right]$ code.

- The dimension $\operatorname{dim}(\mathcal{C})=k$ of $\mathcal{C}$ is the number of symbols in a message stored using the code $\mathcal{C}$, i.e., the dimension of the message space $\mathbb{F}_{q}^{k}$.

- The length $n$ of $\mathcal{C}$ is the length of the codewords in the codebook. We define the redundancy of the code as $n-k$, and the rate of the code as $\frac{k}{n}$.

- The minimum distance $d_{\min }$ of a code is defined as

$$
d_{\min }=\min _{\substack{u, v \in \mathcal{C} \\ u \neq v}} \operatorname{dist}(u, v),
$$

where $\operatorname{dist}(\cdot, \cdot)$ is the distance between two codewords.

There are two distance metrics that will be useful to us, the Hamming distance, and the rank distance.

Definition 1 (Hamming distance). The Hamming distance $d_{H}(\cdot, \cdot)$ between two codewords in a code $\mathcal{C}$ is the number of positions where they differ.

The rank distance utilizes the isomorphism $\mathbb{F}_{q^{s}} \cong \mathbb{F}_{q}^{s}$, which implies a bijective map from vectors $a \in \mathbb{F}_{q^{s}}^{n}$ to matrices $A \triangleq \Phi(a) \in \mathbb{F}_{q}^{s \times n}$. The rank of a vector is defined as the rank of its matrix representation, i.e. $\operatorname{rk}_{q}(a)=\operatorname{rk}_{q}(A)$.

Definition 2 (Rank distance). The rank distance between a and $b$ for $a, b \in$ $\mathbb{F}_{q^{s}}^{n}$ is the rank of the difference of the two matrix representations:

$$
d_{\mathrm{R}}(a, b) \triangleq \operatorname{rk}_{q}(a-b)=\operatorname{rk}_{q}(A-B) .
$$

Any code satisfies a relation between its minimum distance $d_{\min }$ and its redundancy $n-k$. This relation is the Singleton bound.

Proposition 1 (Singleton Bound). For any code $\mathcal{C}$, the redundancy $n-k$ is strictly greater than the minimum distance, i.e.

$$
n-k \geq d_{\min }-1 \text {. }
$$

A generator matrix $G_{\mathcal{C}}$ of an $\left[n, k, d_{\text {min }}\right] \operatorname{code} \mathcal{C}$ is a $k \times n$ matrix, such that $\operatorname{rk}\left(G_{\mathcal{C}}\right)=k$. Then a message $m \in \mathbb{F}_{q}^{k}$ is encoded into a codeword $c$ by the relation

$$
c=m G_{\mathcal{C}}
$$


An information set of an $\left[n, k, d_{\text {min }}\right]$ code $\mathcal{C}$ is defined as any subset $\mathcal{I}_{k}$ of $[n]$, with $\left|\mathcal{I}_{k}\right|=k$, such that the columns of $G_{\mathcal{C}}$ indexed by $\mathcal{I}_{k}$ are linearly independent.

Definition 3. The dual of a code $\mathcal{C} \in \mathbb{F}_{q}$ is the code $\mathcal{C}^{\perp}=\left\{x \in \mathbb{F}_{q} \mid\langle x, c\rangle=\right.$ $0 \forall c \in \mathcal{C}\}$. Moreover, $\operatorname{dim}(\mathcal{C})+\operatorname{dim}\left(\mathcal{C}^{\perp}\right)=n$.

We next define two information theoretic quantities, namely, the entropy and the mutual information [11].

Definition 4 (Entropy). The entropy $H(X)$ of a discrete random variable $X$ is a measure of the uncertainty of $X$. It is defined by

$$
H(X)=-\sum_{x \in X} p(x) \log (p(x))
$$

where $p(x)$ is the probability of event $X=x$.

The conditional entropy of a random variable $Y$ given another random variable $X$ is defined as

$$
H(Y \mid X)=\sum_{x \in X} p(x) H(Y \mid X=x)
$$

Definition 5 (Mututal information). Consider two random variables $X$ and $Y$. The mutual information $I(X ; Y)$ between $X$ and $Y$ is the reduction in the uncertainty of $X$ due to the knowledge of $Y$. It is formally defined as

$$
I(X ; Y)=H(X)-H(X \mid Y) .
$$

\subsection{Maximum Distance Separable (MDS) Codes}

Maximum Distance Separable (MDS) codes are erasure codes that have minimum distance $d_{\min }=n-k+1$, i.e., meet the singleton bound with equality. Those codes are capable of correcting $n-k$ erasures and $\left\lfloor\frac{n-k}{2}\right\rfloor$ errors. We shortly denote the code as an $[n, k]$ MDS code, as $d_{\text {min }}$ is implied by definition. The dual of an $[n, k]$ MDS code is an $[n, n-k]$ MDS code, i.e., the class of MDS codes is closed under the dual operation. Reed-Solomon (RS) codes are the most widely known MDS codes.

A very important property of MDS codes is that any $k$-subset forms an information set, in other words, any $k \times k$ submatrix of its generator matrix $G$ is invertible [29]. 
Example 1. An $[n, k] M D S$ code with $n=k+1$ is a simple parity check code with generator matrix

$$
G=\left(\begin{array}{cc}
I_{k \times k} & \mathbf{1}_{k}
\end{array}\right)
$$

where $\mathbf{1}_{k}$ is the all ones vector of size $k$. For this code, servers $1, \ldots, k$ store the uncoded information symbols and server $k+1$ stores the sum of all the symbols. This code has rate $R_{\mathcal{C}}=\frac{n-1}{n}$, and one can see that any $k \times k$ submatrix of $G$ is invertible. This code has minimum distance $d_{\text {min }}=(k+1)-k+1=2$, and can therefore correct only one erasure.

\subsubsection{Reed-Solomon (RS) codes}

Let us start by defining a Vandermonde matrix.

Definition 6 (Vandermonde matrix). A Vandermonde matrix $V$ is a $k \times n$ matrix with $V_{i j}=\alpha_{j}^{i-1}$, where $\alpha_{1}, \ldots, \alpha_{n}$ are $n$ distinct elements of $\mathbb{F}$, hence $|\mathbb{F}| \geq n$. The Vandermonde matrix is given by the following matrix

$$
V=\left(\begin{array}{ccc}
\alpha_{1}^{0} & \ldots & \alpha_{n}^{0} \\
\vdots & \ddots & \vdots \\
\alpha_{1}^{k-1} & \ldots & \alpha_{n}^{k-1}
\end{array}\right)
$$

Let $k \leq n$ and denote the space of all single-variable polynomials of degree $<k$ by $\mathbb{F}[z]^{<k}$. An $R S[n, k]$ code is defined as

$$
R S[n, k]=\left\{\operatorname{eval}(f(z)): f(z) \in \mathbb{F}[z]^{<k}\right\}
$$

where eval is the map defined as

$$
\text { eval }: \mathbb{F}[z] \rightarrow \mathbb{F}^{n}, \quad \operatorname{eval}(f(z))=\left(f\left(\alpha_{1}\right), \ldots, f\left(\alpha_{n}\right)\right) \in \mathbb{F}^{n} .
$$

Let $G$ be a Vandermonde matrix and $f_{0}, \ldots, f_{k-1}$ be information symbols. We can see that $G$ is a generator matrix of an $[n, k] \mathrm{RS}$ code,

$$
\operatorname{eval}(f(z))=\left(f_{0}, \ldots, f_{k-1}\right) \cdot G
$$

\subsubsection{Reed-Solomon Codes for Storage}

Consider a storage code $\mathcal{C}$ such that $\mathcal{C}=R S[n, k]$. We assume that each file $f^{i}$ is divided into $L$ stripes. We can represent the file $f^{i}$ as an $L \times k$ matrix 


$$
f^{i}=\left(\begin{array}{cccc}
f_{1,0}^{i} & f_{1,1}^{i} & \ldots & f_{1, k-1}^{i} \\
f_{2,0}^{i} & f_{2,1}^{i} & \ldots & f_{2, k-1}^{i} \\
\vdots & \vdots & \vdots & \vdots \\
f_{L, 0}^{i} & x_{L, 1}^{i} & \ldots & x_{L, k-1}^{i}
\end{array}\right)
$$

The $k$ information symbols of row $f_{\ell}^{i}$ are encoded as coefficients of a polynomial

$$
f_{\ell}^{i}(z)=f_{\ell, 0}^{i}+f_{\ell, 1}^{i} z+\cdots+f_{\ell, k-1}^{i} z^{k-1}
$$

of degree $<k$. This polynomial is evaluated at $n$ distinct points $\alpha_{1}, \ldots, \alpha_{n} \in$ $\mathbb{F}_{q}$, and the evaluations of these polynomials at $\alpha_{j}$ are stored on server $j$. Therefore, the matrix $y^{i}=f^{i} \cdot G$ of the encoded symbols of file $f^{i}$ is of the form

$$
y^{i}=\left(\begin{array}{ccc}
f_{1}^{i}\left(\alpha_{1}\right) & \cdots & f_{1}^{i}\left(\alpha_{n}\right) \\
\vdots & \ddots & \vdots \\
f_{L}^{i}\left(\alpha_{1}\right) & \cdots & f_{L}^{i}\left(\alpha_{n}\right)
\end{array}\right)
$$

\subsubsection{Star Product of RS Codes}

We next define the star product of two subvector spaces. We denote the star product defined in [18] as $\star$.

Definition 7. For any two vector spaces $V$ and $W$ in $\mathbb{F}_{q}^{n}$, we can define $V \star W$ to be the subspace of $\mathbb{F}_{q}^{n}$ generated by the Hadamard products, such that $V \star W=\langle v \star w \mid v \in V, w \in W\rangle=\left\langle\left(v_{1} w_{1}, \ldots, v_{n} w_{n}\right) \mid v=\left\{v_{i}\right\}, w=\left\{w_{i}\right\}\right\rangle$.

We cite the most important properties of the star product from [18].

- Let $\mathcal{R}(n)_{q} \subseteq \mathbb{F}_{q}^{n}$ be the repetition code of length $n$ over $\mathbb{F}_{q}$. For any linear code $\mathcal{C} \subseteq \mathbb{F}_{q}^{n}$, the star product $\mathcal{C} \star \mathcal{R}(n)_{q}=\mathcal{C}$.

- For any MDS code, $\mathcal{C} \subseteq \mathbb{F}_{q}^{n},\left(\mathcal{C} \star \mathcal{C}^{\perp}\right)^{\perp}=\mathcal{R}(n)_{q}$.

- The star product of two RS codes in $\mathbb{F}_{q}^{n}$ is again an RS code. More specifically, let $\mathcal{C}=R S[n, k]$ and $\mathcal{D}=R S\left[n, k^{\prime}\right]$ be two RS codes with the same evaluation points $\alpha_{1}, \ldots, \alpha_{n}$, then

$$
\begin{aligned}
\mathcal{C} \star \mathcal{D}=\left\langle\left\{\left(h\left(\alpha_{1}\right), h\left(\alpha_{2}\right), \ldots, h\left(\alpha_{n}\right)\right): h(z)\right.\right. & =f(z) g(z), \\
f(z) & \left.\left.\in \mathbb{F}[z]^{<k}, g(z) \in \mathbb{F}[z]^{<k^{\prime}}\right\}\right\rangle,
\end{aligned}
$$

which is an $R S\left[n, k+k^{\prime}-1\right]$ code, as the degree of the polynomial $h(z)$ is $\operatorname{deg}(h)=\operatorname{deg}(f)+\operatorname{deg}(g)=k-1+k^{\prime}-1=k+k^{\prime}-2$. 


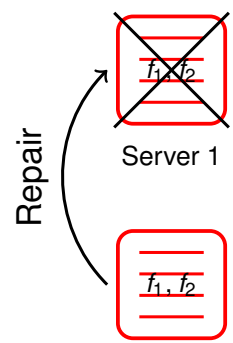

Server 2

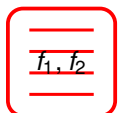

Server 3

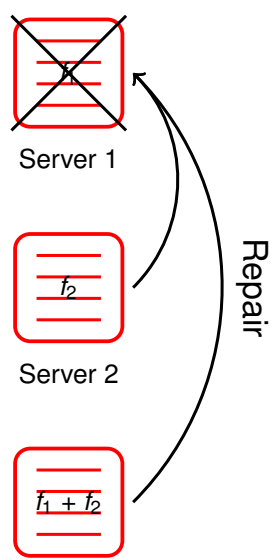

Server 3

$[3,1]$ replicated system

$[3,2]$ parity-check coded DSS

Figure 2.1. A replicated system (left) vs an MDS coded system (right) on three servers.

We will compare the essential features of a $[3,2]$ MDS coded DSS to those of a [3, 1] replicated system in Example 2.

Example 2. Assume we want to store $m$ files $f^{1}, \ldots, f^{m}$ reliably on three servers, and each file is a pair $f^{i}=\left(f_{1}^{i}, f_{2}^{i}\right)$. One way to do that is to store both parts of all $m$ files on each server. Another way is to use a [3,2] MDS code, where the first server stores the first part $f_{1}=\left\{f_{1}^{i}: i \in[m]\right\}$ of each file, the second server stores the second part $f_{2}=\left\{f_{2}^{i}: i=[m]\right\}$, and the third server stores the sum $f_{1}+f_{2}=\left\{f_{1}^{i}+f_{2}^{i}: i \in[m]\right\}$. The two storage systems are shown in Figure 2.1.

In this example, one can see the tradeoff between the storage overhead (or code rate) and the fault tolerance of the code. The code rate of the replicated system is $1 / 3$, while the code rate of the parity-check coded system is $2 / 3$. Therefore, the storage overhead of a parity check coded DSS is lower than that of a replicated system. On the other hand, the replicated system can tolerate two server failures while the MDS coded system can tolerate only one server failure. In other words, two servers are needed to repair a failed node in a [3,2] parity-check coded system, while only one is needed for repair in a $[3,1]$ replicated system, as shown in Figure 2.1. Moreover, in a replicated system, the repair is a repair by transfer, i.e., no computation is needed, whereas for a parity-check coded system, one needs to subtract the two entries to get the contents of a replacing node. 


\subsection{Regenerating Codes}

Another subclass of erasure codes is the class of regenerating codes. A regenerating code with the set of parameters $(n, k, d, B, \alpha, \beta)$ is a code where a set of files of size $B$ each are encoded, such that $\alpha$ coded symbols from each file are stored on each of the $n$ servers, and any set of $k$ servers can reconstruct a file. An important property of this code is the fact that any $d \geq k$ servers are sufficient for repair of a failed server by downloading $\beta$ symbols from each. The total repair bandwidth is then $\gamma=d \beta$. It is important to note here that $d$ is not equivalent to the minimum distance of the code, $d_{\text {min }}$.

Dimakis et. al. prove in [14] that any storage (erasure) code with the above property satisifes the cut-set bound given in (2.4) with equality.

Definition 8 (Cut-set bound). The cut-set bound is an upper bound on the file size B:

$$
B \leq \sum_{i=0}^{k-1} \min \{\alpha,(d-i) \beta\} .
$$

It is also shown that equality in (2.4) defines a tradeoff between the parameters $\alpha$ and $\gamma=\beta d$, which cannot be simultaneously minimized. Codes lying on this tradeoff curve are called regenerating codes, i.e. regenerating codes meet the bound (2.4) with equality. There are two extremal points on the tradeoff curve defined by (2.4): the best storage efficiency, which is referred to as the minimum-storage regenerating (MSR) point, and the best repair bandwidth, which is referred to as the minimumbandwidth regenerating (MBR) point. Regenerating codes minimizing $\gamma=\beta d$ achieve the MBR point, while those minimizing $\alpha$ attain the MSR point. In [33], the authors discuss regenerating codes on the MBR point and on the MSR point, and construct codes that achieve those two extremal points. Let us take a closer look at these codes.

\subsubsection{Product-Matrix (PM) Constructions}

One of the constructions of regenerating codes was introduced by Rashmi et al. in [33]. These constructions use the product-matrix (PM) framework. To simplify the construction, the authors set $\beta=1$ without loss of generality. This assumption is easily generalizable to the case where $\beta \neq 1$ by striping the files. In all what follows in this section, we assume $\beta=1$. 


\subsubsection{PM Codes in the MBR Setting [33]}

At the MBR point, we optimize $\gamma=\alpha$. We can then see that in this setting, $d=d \beta=\gamma=\alpha$. Therefore, in this setting, the following constraints need to hold [14]:

$$
\alpha=d \quad \text { and } \quad B=k(d-k)+\frac{k(k+1)}{2} .
$$

Each file $f^{i}$ is divided into $B$ symbols, that are arranged in a $d \times d$ matrix $\mathbf{M}^{i}$ such that

$$
\mathbf{M}^{i}=\left(\begin{array}{cc}
\mathbf{S} & \mathbf{T} \\
\mathbf{T}^{\top} & \mathbf{0}
\end{array}\right)
$$

The submatrix $\mathbf{S}$ is a $k \times k$ symmetric matrix containing $\frac{k(k+1)}{2}$ distinct file symbols, and $\mathbf{T}$ is a $k \times(d-k)$ matrix containing the remaining $k(d-k)$ file symbols.

We will refer to this construction as a PM-MBR code, and denote it by $\mathcal{C}$. Notice that $\mathcal{C}$ is an $[n d, B]$ linear code over $\mathbb{F}_{q}$. We cite an example given by the authors in [33, Sec. IV.A.].

Example 3 (PM-MBR code). Consider the setting $(n, k, d)=(6,3,4)$ over the field $\mathbb{F}_{7}$. The original file contains $B=k(d-k)+\frac{k(k+1)}{2}=9$ symbols. In this example, $\alpha=d=4$, thus, every server stores 4 encoded symbols from each file. The code rate for this example is $R_{\mathcal{C}}=\frac{B}{n \alpha}=3 / 8$. The generator and the message matrices are given as:

$$
G=\left(\begin{array}{llllll}
1 & 1 & 1 & 1 & 1 & 1 \\
1 & 2 & 3 & 4 & 5 & 6 \\
1 & 4 & 2 & 2 & 4 & 1 \\
1 & 1 & 6 & 1 & 6 & 6
\end{array}\right) \text { and } \mathbf{M}^{i}=\left(\begin{array}{cccc}
f_{1}^{i} & f_{2}^{i} & f_{3}^{i} & f_{7}^{i} \\
f_{2}^{i} & f_{4}^{i} & f_{5}^{i} & f_{8}^{i} \\
f_{3}^{i} & f_{5}^{i} & f_{6}^{i} & f_{9}^{i} \\
f_{7}^{i} & f_{8}^{i} & f_{9}^{i} & 0
\end{array}\right)
$$

For instance, the third server stores the four symbols

$$
\begin{aligned}
& f_{1}^{i}+3 f_{2}^{i}+2 f_{3}^{i}+6 f_{7}^{i}, \\
& f_{2}^{i}+3 f_{4}^{i}+2 f_{5}^{i}+6 f_{8}^{i}, \\
& f_{3}^{i}+3 f_{5}^{i}+2 f_{6}^{i}+6 f_{9}^{i}, \\
& f_{7}^{i}+3 f_{8}^{i}+2 f_{9}^{i}
\end{aligned}
$$

from file $f^{i}$. 


\subsubsection{PM Codes in the MSR Setting}

At the MSR point, we minimize storage per server, thus, $\alpha=\frac{B}{k}$. Therefore, the parameters $\alpha$ and $B$ are given by:

$$
\alpha=\frac{B}{k}=d-k+1 \quad \text { and } \quad B=k(d-k+1) .
$$

The authors in [33] construct PM codes in the MSR setting for $d \geq 2 k-2$. In this setting, $d \leq 2 \alpha$ and $B \leq \alpha(\alpha+1)$. In this work, for simplicity, we assume the case where $d=2 k-2$ as it is the case for the first construction given in [33]. Thus, $d$ and $B$ can be simplified as $d=2 \alpha$ and $B=\alpha(\alpha+1)$.

File symbols are arranged in a $2 \alpha \times \alpha$ matrix

$$
\mathbf{M}^{i}=\left(\begin{array}{l}
\mathbf{S}_{1} \\
\mathbf{S}_{2}
\end{array}\right)
$$

where $\mathbf{S}_{1}$ and $\mathbf{S}_{2}$ are $\alpha \times \alpha$ symmetric matrices containing $\frac{\alpha(\alpha+1)}{2}$ file symbols each. Let $G$ be a $2 \alpha \times n$ Vandermonde matrix over $\mathbb{F}_{q}$. As in the MBR setting, the $j$-th column of a codeword $y^{i}=\mathbf{M}^{i \top} G$ is stored on server $j$, for $j \in[n]$.

We refer to this construction as a PM-MSR code. We rewrite the example given in [33, Sec. V.A.].

Example 4 (PM-MSR code). Consider the setting $(n, k, d)=(6,3,4)$ over $\mathbb{F}_{13}$, which gives the file size $B=6$. Every server is storing 2 coded symbols from each file and the code rate is $\frac{B}{n \alpha}=1 / 2$. Matrices $G$ and $\mathbf{M}^{i}$ are then given by

$$
G=\left(\begin{array}{cccccc}
1 & 1 & 1 & 1 & 1 & 1 \\
1 & 2 & 3 & 4 & 5 & 6 \\
1 & 4 & 9 & 3 & 12 & 10 \\
1 & 8 & 1 & 12 & 8 & 8
\end{array}\right) \text {, and } \mathbf{M}^{i}=\left(\begin{array}{cc}
f_{1}^{i} & f_{2}^{i} \\
f_{2}^{i} & f_{3}^{i} \\
f_{4}^{i} & f_{5}^{i} \\
f_{5}^{i} & f_{6}^{i}
\end{array}\right) .
$$

For example, server 3 is storing the two coded symbols $f_{1}^{i}+3 f_{2}^{i}+9 f_{4}^{i}+f_{5}^{i}$ and $f_{2}^{i}+3 f_{3}^{i}+9 f_{5}^{i}+f_{6}^{i}$ from file $f^{i}$.

\subsection{Network Coding}

A network is a channel consisting of source nodes, receiver nodes, and some intermediate nodes. The intermediate nodes forward the received messages. If multiple messages arrive at a single intermediate node at the same time, the node will forward a linear combination of the messsages to 
the next layer of nodes $[1,21,24,31]$. In this work, we concentrate on the case where one source, namely the user, wants to retrieve a file from a DSS consisting of a set of sinks, namely the servers. The user transmits data over a random linear network, and along the way, erroneous messages may be inserted on any edge of the network. A network model is shown in Figure 2.2.

Assume the sender wants to transmit $n$ messages to the receiver, organized as the rows of a matrix $Q \in \mathbb{F}_{q^{s}}^{n \times m}$. Note that rank codes are defined here as subsets of an $n$-dimensional vector space over an extension field $\mathbb{F}_{q^{s}}$. After being transmitted through the random linear network, the receiver gets the matrix $R \in \mathbb{F}_{q^{s}}^{n \times m}$, such that

$$
R=A Q+N_{e},
$$

where $A \in \mathbb{F}_{q^{s}}^{n \times n}$ denotes the channel matrix, $N_{e} \in \mathbb{F}_{q^{s}}^{n \times m}$ denotes the overall error vector. We assume, for simplicity, that the field size of the entries of the network matrices is equal to $q^{s}$, i.e., the linear combinations have coefficients in $\mathbb{F}_{q^{s}}$. We also assume the random channel matrix $A$ to be a square matrix.

The probability that a square matrix is full rank is given in the following proposition.

Proposition 2. (Probability of full rank, [21]) Let $A \in \mathbb{F}_{q}^{\kappa \times \kappa}$ be a square matrix with elements chosen uniformly at random from $\mathbb{F}_{q}$. Then

$$
P=\mathbb{P}\left(\mathrm{rk}_{q}(A)=\kappa\right) \geq\left(1-\frac{1}{q}\right)^{\kappa} .
$$

Network coding has many uses [14], for instance, it increases network capacity, improves robustness, improves distributed compression, etc...

\subsection{Maximum Rank Distance (MRD) Codes}

A linear rank-metric code is a linear subspace of $\mathbb{F}_{q^{s}}^{n}$ with dimension $k$ and minimum rank distance $d_{\min }$ and is denoted by $\left[n, k, d_{\min }\right]_{q}^{\mathrm{R}}$. For linear codes with $n \leq s$, the Singleton bound in Definition 1 applies, with the distance being calculated as the rank distance, thus, $d_{\min } \leq n-k+1$ $[12,19]$. Codes achieving this bound with equality, i.e., $d_{\min }=n-k+1$, are called maximum rank distance (MRD) codes.

A special class of MRD codes are Gabidulin codes [19]. A Gabidulin code $\mathcal{G}(n, k)$ over $\mathbb{F}_{q^{s}}$ of length $n \leq s$ and dimension $k<n$ is defined by 


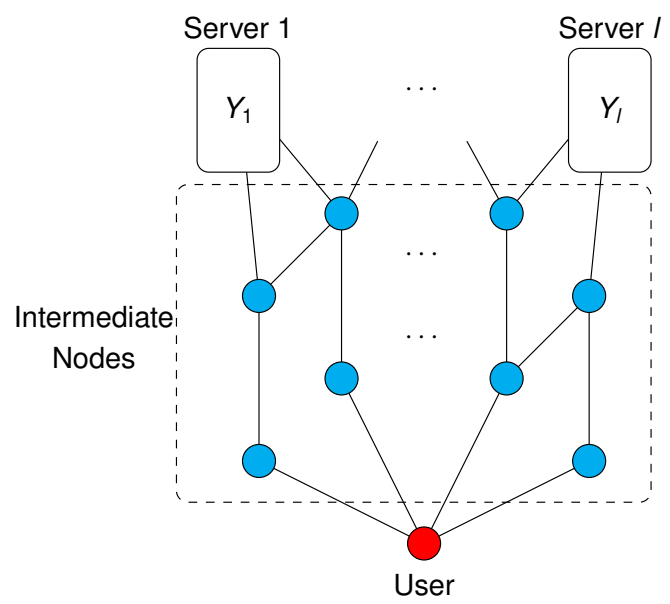

Figure 2.2. Network Model: The user is connected to the servers via intermediate nodes. When data is sent from the user to the servers, or from server to user, the messages go through a random network using the intermediate nodes.

evaluating degree-restricted linearized polynomials:

$$
\mathcal{G}(n, k)=\left\{\left(f\left(\alpha_{0}\right), f\left(\alpha_{1}\right), \ldots, f\left(\alpha_{n-1}\right)\right): f(z)=\sum_{i=0}^{k-1} f_{i} z^{q^{i}}\right\},
$$

where $\alpha_{0}, \alpha_{1}, \ldots, \alpha_{n-1} \in \mathbb{F}_{q^{s}}$ have to be linearly independent over $\mathbb{F}_{q}$. Gabidulin codes are MRD codes, i.e., $d=n-k+1$, cf. [19, 35]. Gabidulin codes are a natural analog of RS codes.

As in the case of RS codes, Gabidulin codes can be used for storage. Let $\mathcal{C}$ be a storage code such that $\mathcal{C}=\mathcal{G}(n, k)$. Each file $f^{i}$ can be represented by an $L \times K$ matrix as in (2.1). The information symbols of row $f_{\ell}^{i}$ of file $f^{i}$ are encoded as coefficients of the polynomial $f(z)$. Thus the matrix $y^{i}$ of encoded symbols is of the same form as in (2.3).

\subsubsection{Star Product of Gabidulin Codes}

We denote the star product in the context of Gabidulin codes as $\star_{G a b}$ which is defined in [30] and Publication VI.

Definition 9. For any two vectors $v$ and $w$ in $\mathbb{F}_{q^{s}}^{n}$, and let $V=\Phi(v)$ and $W=\Phi(w)$ be their matrix representations in $\mathbb{F}_{q}^{s \times n}$. Recall that $\Phi$ is the bijective map from vectors in $\mathbb{F}_{q^{s}}^{n}$ to matrices in $\mathbb{F}_{q}^{s \times n}$. We define the star product $v \star_{G a b} w=V^{\top} W$.

We next define the star product more specifically for Gabidulin codes.

Let $\alpha_{1}, \ldots, \alpha_{n} \in \mathbb{F}_{q^{s}}$ be linearly independent over $\mathbb{F}_{q}$ which implies $n \leq s$. Let $\mathcal{C}$ and $\mathcal{D}$ be two Gabidulin codes such that $\mathcal{C}$ is a $\mathcal{G}(n, k)$ Gabidulin code

$$
\mathcal{C}=\left\{\left(f\left(\alpha_{0}\right), f\left(\alpha_{1}\right), \ldots, f\left(\alpha_{n-1}\right)\right): f(z)=f_{0} z^{q^{0}}+f_{1} z^{q^{1}}+\cdots+f_{k-1} z^{q^{k-1}}\right\} .
$$


and $\mathcal{D}$ is a $\mathcal{G}\left(n, k^{\prime}\right)$ Gabidulin code

$\mathcal{D}=\left\{\left(g\left(\alpha_{0}\right), g\left(\alpha_{1}\right), \ldots, g\left(\alpha_{n-1}\right)\right): g(z)=g_{0} z^{q^{0}}+g_{1} z^{q^{1}}+\cdots+g_{k^{\prime}-1} z^{q^{k^{\prime}-1}}\right\}$.

The star product of $\mathcal{C}$ and $\mathcal{D}$ is then defined by:

$$
\begin{aligned}
\mathcal{C} \star_{G a b} \mathcal{D}=\left\langle\left\{\left(h\left(\alpha_{0}\right), h\left(\alpha_{1}\right), \ldots, h\left(\alpha_{n-1}\right)\right): h(z)\right.\right. & =f(g(z)), \\
f(z)=\sum_{i=0}^{k-1} f_{i} z^{q^{i}}, g(z) & \left.\left.=\sum_{i=0}^{k-1} g_{i} z^{q^{i}}\right\}\right\rangle,
\end{aligned}
$$

which is a $\mathcal{G}\left(n, k+k^{\prime}-1\right)$ Gabidulin code.

Example 5. Consider a storage system over $\mathbb{F}_{2^{5}}$ with primitive element $\alpha$ and primitive polynomial $z^{5}+z^{2}+1$. Let $\mathcal{C}$ be a $\mathcal{G}(5,3)$ Gabidulin code defined by:

$$
\mathcal{C}=\left\{\left(f(1), f(\alpha), f\left(\alpha^{2}\right), f\left(\alpha^{3}\right), f\left(\alpha^{4}\right)\right): f(z)=f_{0} z+f_{1} z^{2}+f_{2} z^{4}\right\},
$$

and $\mathcal{D}$ be a $\mathcal{G}(5,2)$ Gabidulin code defined by:

$$
\mathcal{D}=\left\{\left(g(1), g(\alpha), g\left(\alpha^{2}\right), g\left(\alpha^{3}\right), g\left(\alpha^{4}\right)\right): g(z)=g_{0} z+g_{1} z^{2}\right\}
$$

Now the code $\mathcal{C} \star \mathcal{D}$ is a $\mathcal{G}(5,4)$ Gabidulin code defined by:

$$
\begin{aligned}
\mathcal{C} \star \mathcal{D}=\left\langle\left\{\left(h(1), h(\alpha), h\left(\alpha^{2}\right), h\left(\alpha^{3}\right), h\left(\alpha^{4}\right)\right): h(z)\right.\right. & =f(g(z)) \\
& \left.\left.=\sum_{i=0}^{2} \sum_{j=0}^{1} f_{i}\left(g_{j}\right) z^{2^{i+j}}\right\}\right\rangle .
\end{aligned}
$$




\section{Private Information Retrieval (PIR)}

\subsection{Definition}

Private information Retrieval (PIR) is a problem introduced by Chor et. al. in [10], in which the user is allowed to retrieve a file from a distributed storage system (DSS), without revealing the identity of the desired file to the servers. Of course, a naïve solution to achieve this is to download all the files in the system, but that would incur a high communication complexity. Two settings of PIR have been studied in the literature:

- Computational PIR (CPIR), where the identity of the desired file is computationally hard to find given the query received by the server. In this setting, privacy is achieved under certain hardness assumptions and assuming limited computational power at the server. Some work has been done to achieve CPIR on single server storage,without downloading all the files in the system [27, 8].

- Information-Theoretic PIR (IT-PIR) where no limit on the servers' computational power is assumed. In this setting, it was proved in [10] that for a single server setting, the user has to download all the files to achieve PIR. This thesis focuses only on IT-PIR, therefore, in what follows, PIR scheme is used to denote an IT-PIR scheme.

The original scheme by Chor et. al. considered a DSS with two servers on which a data vector $\mathbf{f}=\left[\begin{array}{llll}f^{1} & f^{2} & \ldots & f^{m}\end{array}\right]^{\top}$ consisting of $m$ files is replicated. Let us first assume that the user is not concerned with being private. In that case, if a user wants to retrieve a file $f^{\nu}, \nu \in\{1, \ldots, m\}$, she will send the deterministic vector $\mathbf{e}_{\nu}$, consisting of all zeros and a single one on position $\nu$ to one of the servers. The server projects this deterministic vector on its stored data, which gives the desired file $\left\langle\mathbf{f}, \mathbf{e}_{\nu}\right\rangle=f^{\nu}$, 


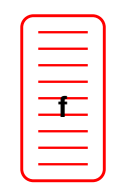

Server 1

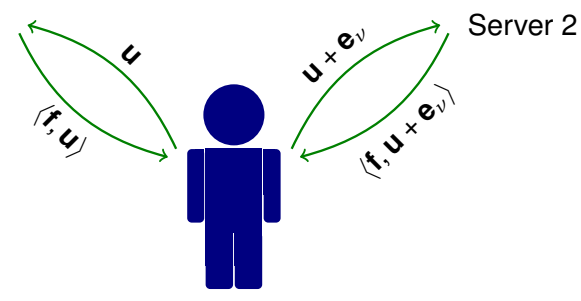

$$
\left\langle\mathbf{f}, \mathbf{u}+\mathbf{e}_{\nu}\right\rangle-\langle\mathbf{f}, \mathbf{u}\rangle=\left\langle\mathbf{f}, \mathbf{e}_{\nu}\right\rangle=f^{\nu}
$$

Figure 3.1. Chor's scheme on 2 -server replicated system

and sends it back to the user. On the other hand, if the user wants to keep the file identity $\nu$ hidden from the servers, the user can generate a random vector $\mathbf{u} \in \mathbb{F}_{q}^{m}$, and send it to one of the servers, then add the deterministic vector $\mathbf{e}_{\nu}$ to the random vector $\mathbf{u}$ and send $\mathbf{u}+\mathbf{e}_{\nu}$ to the other server, as shown in Figure 3.1. One can see that each of those query vectors is random from the servers' point of view. From the responses of those queries, the user receives the product $\langle\mathbf{f}, \mathbf{u}\rangle$ and $\left\langle\mathbf{f}, \mathbf{u}+\mathbf{e}_{\nu}\right\rangle$, from which, she can retrieve the product $\left\langle\mathbf{f}, \mathbf{e}_{\nu}\right\rangle=f^{\nu}$.

\subsection{Parameters of a PIR Scheme}

In the basic scheme example described in Section 3.1, one can see that if the two servers communicate their received queries, privacy is lost, since the servers can then recover the deterministic vector $\mathbf{e}_{\nu}$ by subtracting the two received queries. In the original Chor scheme, the servers are assumed not to collude. In general, servers in a DSS might communicate in an effort to figure out the file identity $\nu$ of the user's desired file. A PIR scheme under the assumption that up to $t$ servers might communicate in a storage system is called a $t$-private PIR scheme, and is defined formally in Definition 10.

Definition 10 (t-collusion). A PIR scheme protects against $t$-collusion, or is $t$-private, if for every subset of servers $T=\left\{j_{1}, \ldots, j_{t}\right\} \subseteq\{1, \ldots, n\}$ of size $|T|=t$, the mutual information between the desired file index $\nu$ and the queries to all t servers, $q_{j_{1}}, \ldots, q_{j_{t}}$, should be zero, i.e.,

$$
I\left(\nu ; q_{j_{1}}, \ldots, q_{j_{t}}\right)=0 .
$$


In order to retrieve a file privately, the user needs to communicate more data symbols with the servers than is needed to retrieve a file without the privacy concern. In the classical setting of PIR, the communication complexity of a PIR scheme constitutes of both the upload and the download communication. For instance, in the basic scheme example given in Section 3.1, the user uploads two vectors of length $m$ and downloads 2 symbols in order to retrieve $f^{\nu}$ privately.

In current storage systems and the information-theoretic reformulation of the PIR problem, the size of the files is assumed to be much larger than the size of the query vector, [9]. Under this assumption, the download cost is much more significant than the upload cost in the communication complexity. Therefore, in recent literature on PIR, the download communication complexity constitutes the efficiency of a PIR scheme.

Definition 11 (PIR Rate). The metric of efficiency of a PIR scheme is the PIR rate, denoted $R_{P I R}$. The PIR rate is defined as the ratio of the size of the desired file to the amount of total downloaded data.

The PIR capacity, denoted by $C_{P I R}$, is the supremum of all achievable $P I R$ rates for a certain setting.

Originally, PIR schemes were constructed on storage systems where the files are replicated on $n$ servers, i.e., where the storage code $\mathcal{C}$ is the repetition code $\mathcal{R}(n)_{q}[2,5,6,7,13,16,38,45]$. The capacity of a $t$-private PIR scheme over a storage system containing $m$ files replicated on $n$ servers was found in [39] to be

$$
C_{P I R}=\frac{1-\frac{t}{n}}{1-\left(\frac{t}{n}\right)^{m}} .
$$

On the other hand, with the increased use of DSSs with coded storage, a lot of work was done to construct PIR schemes over coded storage systems, and to quantify the PIR capacity in those settings. PIR schemes on coded storage were first studied as an application of batch codes in [22]. More recently, constructing PIR schemes on DSSs that use erasure codes was studied in [36], in which the authors show that downloading one extra bit is sufficient to achieve PIR when the number of servers $n$ is assumed to be exponential in the number of files $m$. Tradeoffs between the storage cost and the download cost for PIR schemes over coded storage systems were found in [9]. Afterwards, the PIR capacity for a coded DSS using an $[n, k]$ MDS code was found to be

$$
C_{P I R}=\frac{1-\frac{k}{n}}{1-\left(\frac{k}{n}\right)^{m}}
$$




\begin{tabular}{|c|c|c|c|c|c|c|}
\hline Server 1 & Server 2 & $\ldots$ & Server $k$ & Server $k+1$ & $\ldots$ & Server $n$ \\
\hline$f_{1,0}^{1}$ & $f_{1,1}^{1}$ & $\ldots$ & $f_{1, k-1}^{1}$ & $\sum_{i=0}^{k-1} \lambda_{i, k+1} f_{1, i}^{1}$ & $\ldots$ & $\sum_{i=0}^{k-1} \lambda_{i, n} f_{1, i}^{1}$ \\
$\vdots$ & $\vdots$ & $\vdots$ & $\vdots$ & $\vdots$ & $\vdots$ & $\vdots$ \\
$f_{L, 0}^{1}$ & $f_{L, 1}^{1}$ & $\ldots$ & $f_{L, k-1}^{1}$ & $\sum_{i=0}^{k-1} \lambda_{i, k+1} f_{L, i}^{1}$ & $\ldots$ & $\sum_{i=0}^{k-1} \lambda_{i, n} f_{L, i}^{1}$ \\
\hline$\vdots$ & $\vdots$ & $\vdots$ & $\vdots$ & $\vdots$ & $\vdots$ & $\vdots$ \\
\hline$f_{1,0}^{m}$ & $f_{1,1}^{m}$ & $\ldots$ & $f_{1, k-1}^{m}$ & $\sum_{i=0}^{k-1} \lambda_{i, k+1} f_{1, i}^{1}$ & $\ldots$ & $\sum_{i=0}^{k-1} \lambda_{i, n} f_{1, i}^{m}$ \\
$\vdots$ & $\vdots$ & $\vdots$ & $\vdots$ & $\vdots$ & $\vdots$ & $\vdots$ \\
$f_{L, 0}^{m}$ & $f_{L, 1}^{m}$ & $\ldots$ & $f_{1, k-1}^{m}$ & $\sum_{i=0}^{k-1} \lambda_{i, k+1} f_{L, i}^{m}$ & $\ldots$ & $\sum_{i=0}^{k-1} \lambda_{i, n} f_{L, i}^{1}$ \\
\hline
\end{tabular}

Figure 3.2. The layout of the encoded symbols of the $m$ files in a DSS.

in [4], where the authors construct an achievability scheme on a DSS consisting of a finite number of files, in which each file symbol is subdivided into $n^{m}$ stripes. The capacity of $t$-private PIR schemes on a coded DSS where $t>1$ is still an open problem.

Moreover, PIR schemes over coded storage with arbitrary linear storage codes were constructed in [25], and it was shown that the MDS-PIR capacity can be achieved by some non-MDS codes [28].

\subsection{PIR Schemes on Maximum Distance Separable (MDS) Codes}

In Publication I, we construct a scheme that achieves PIR from an $[n, k]$ MDS coded DSS storing $m$ files, where $m$ is asymptotically large. WLOG, the DSS is assumed to be encoded using a systematic MDS code $\mathcal{C}$ for simplicity ${ }^{1}$. The storage model is shown in Figure 3.2, and the generator matrix $G_{\mathcal{C}}$ of this storage code $\mathcal{C}$ is given by

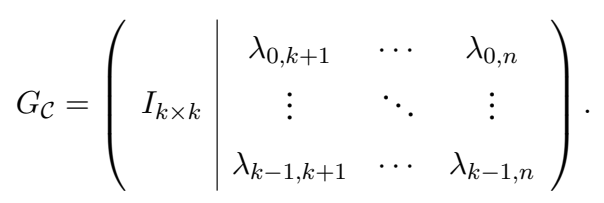

The constructed scheme applies similar techniques as the basic scheme of Chor et al., i.e., hiding the deterministic vectors with a key, similarly to a one-time pad. In this scheme, we assume that every file symbol is subdivided into $L$ stripes, thus every file $f^{i}, i=1, \ldots, m$ can be seen as an $L \times k$ matrix as in (2.1).

To construct a 1-private PIR scheme from the DSS depicted in Figure 3.2, the user generates a random vector $\mathbf{u} \in \mathbb{F}_{\mathbf{q}}^{\mathrm{mL}}$ and encodes it using a repetition code $\mathcal{R}(n)_{q}$. We denote by $D$ the $m L \times n$ random matrix formed

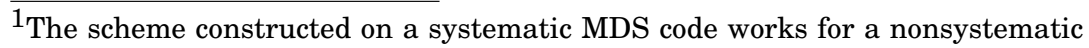
MDS code, but we discuss systematic MDS coded data for simplicity.
} 
by this encoding. As stated in Definition 2.2.3, the star product

$$
\mathcal{C} \star \mathcal{R}(n)_{q}=\mathcal{C}
$$

for any $\left[n, k, d_{\text {min }}\right]$ linear code $\mathcal{C}$. Now, assume the user sends the $j^{\text {th }}$ column of matrix $D$ to server $j$. Then, the server will project the query on its stored data, and send back the projection of the query with their stored data. Using 3.4, we can see that the responses from the servers to the random query matrix $D$ are codewords in code $\mathcal{C}$. Therefore, the responses live in a code with minimum distance $d_{\min }=n-k+1$, which means the response vector can tolerate up to $n-k$ erasures. This implies that if the user adds a 1 to $n-k$ positions of the random vectors, i.e., adding a deviation to $n-k$ positions of the response matrix, the user can then correctly decode those $n-k$ deviations in the response vectors.

From the above discussion, it is clear that the user can send the query matrix $Q^{(i)}$ formed by the sum of the random matrix and the deterministic matrix consisting of $n-k$ ones in different columns to retrieve $n-k$ symbols of the desired file in every query.

Since the user can retrieve $n-k$ symbols per query, the scheme requires the file to be subdivided into $L=\frac{\operatorname{LCM}(k, n-k)}{k}$ stripes, and the user to query $S=\frac{\operatorname{LCM}(k, n-k)}{n-k}$ times for divisibility purposes. If the user retrieves $n-k$ distinct and new symbols in each sub-query, the number of distinct retrieved symbols after $S$ sub-query rounds from the desired file would be $S(n-k)$. Since $L k=S(n-k)$, the user will therefore retrieve all $L k$ stripes of the desired file after $S$ sub-queries.

This is explained in more detail and an explicit construction of the subqueries is given in the proof of Theorem 1 in Publication I. With this construction, we achieve the asymptotic PIR capacity on an MDS coded storage as $m \rightarrow \infty$, that is,

$$
\lim _{m \rightarrow \infty} C_{P I R}=1-\frac{k}{n}
$$

This is stated in Theorem 1, and proved as a construction of the scheme in detail in Section IV in Publication I. We give here a small example.

Example 6. Consider a DSS with $n=4$ servers storing $m$ files, $f^{1}, \ldots, f^{m}$, such that $f^{i}=\left(f_{0}^{i}, f_{1}^{i}\right)$, where $f_{0}^{i}$ and $f_{1}^{i} \in G F\left(3^{\ell}\right)$ for all $i \in\{1, \ldots, m\}$. The files are stored using an $[n, k]=[4,2] M D S$ code over $G F(3)$. Let $A=$ $\left[\begin{array}{llll}f_{0}^{1} & f_{0}^{2} & \ldots & f_{0}^{m}\end{array}\right]^{T}$ and $B=\left[\begin{array}{llll}f_{1}^{1} & f_{1}^{2} & \ldots & f_{1}^{m}\end{array}\right]^{T}$.

Consider the storage system such that servers $1, \ldots, 4$ store $A, B, A+$ $B, A+2 B$, respectively. The user wants to retrieve the file $f^{\nu}=\left(f_{0}^{\nu}, f_{1}^{\nu}\right)$, 


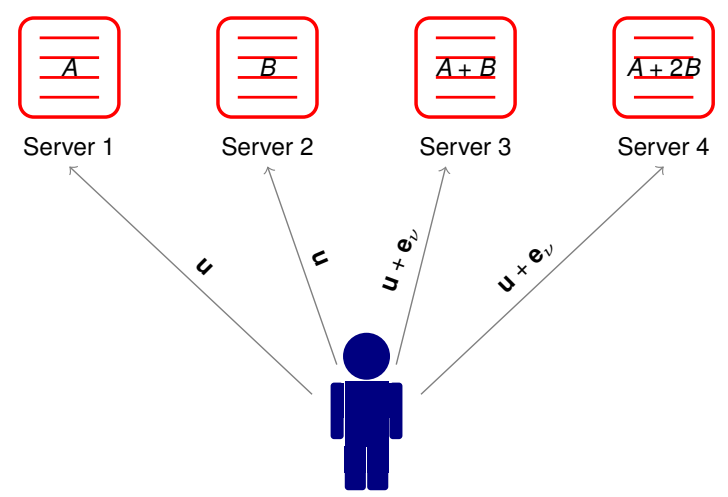

Figure 3.3. The queries sent to the servers in Example 6 .

$\nu \in\{1, \ldots, m\}$, by querying the four servers, without revealing any information about the file index $\nu$ to any of them. Based on the above discussion, the files are subdivided into $L=1$ stripe and the user needs to send $S=1$ query to retrieve the desired file. The user generates a random vector $\mathbf{u}=\left[\begin{array}{lll}u_{1} & \ldots & u_{m}\end{array}\right]^{T}$ with elements chosen uniformly at random from $G F(3)$ and independently of $\nu$. The user then forms the deterministic vector $\mathbf{e}_{\nu}=\left[\begin{array}{lll}\mathbf{0}_{\nu-1} & 1 & \mathbf{0}_{m-\nu}\end{array}\right]^{T}$. Afterwards, the user sends the query vectors $\mathbf{u}$ to servers 1 and 2 and $\mathbf{u}+\mathbf{e}_{\nu}$ to servers 3 and 4 , as shown in Figure 3.3.

Each node responds by projecting its data onto the query vector it receives. Therefore, the responses of servers $1, \ldots, 4$ are given by $\mathbf{u}^{T} A, \mathbf{u}^{T} B$, $\mathbf{u}^{T} A+\mathbf{u}^{T} B+f_{0}^{\nu}+f_{1}^{\nu}, \mathbf{u}^{T} A+2 \mathbf{u}^{T} B+f_{0}^{\nu}+2 f_{1}^{\nu}$, respectively. From the responses of the servers, the user will be able to obtain privately the file $f^{\nu}=\left(f_{0}^{\nu}, f_{1}^{\nu}\right)$. To retrieve the 2 desired file symbols, $f_{0}^{\nu}$ and $f_{1}^{\nu}$, the user needs to download 4 symbols implying $R_{P I R}=2 / 4=1 / 2$, which is asymptotically optimal (as $m \rightarrow \infty)[4,9]$.

For successful decoding of the PIR scheme in Publication I, the user needs to wait for the responses of all the servers to be able to decode the file. However, this may not be possible in many cases due to some servers being unresponsive or due to network failures. Even when all the servers are responsive, some of them may be slow (due to being busy or due to a slow connection). A single slow server will delay the user, even if all the other servers are fast. Therefore, it may be better for the user to "cut" the communication with the slow server and consider it unresponsive. Then, the user can send new queries to the faster servers. In Publication II, we are interested in constructing PIR schemes that have this adaptive property.

A PIR scheme that can work even in the presence of unresponsive servers 


\begin{tabular}{ccccccc} 
& $\overbrace{\text { Stage 1 }}$ & \multicolumn{4}{c}{ Stage 2 } \\
Server 1 & $\mathbf{u}$ & $?$ & $\mathbf{v}$ & $\mathbf{v}+\mathbf{e}_{\nu}$ & $\mathbf{v}+\mathbf{e}_{\nu}$ \\
Server 2 & $\mathbf{u}$ & $\mathbf{v}$ & $?$ & $\mathbf{v}$ & $\mathbf{v}$ \\
Server 3 & $\mathbf{u}+\mathbf{e}_{\nu}$ & $\mathbf{v}+\mathbf{u}$ & $\mathbf{v}+\mathbf{u}$ & $?$ & $\mathbf{v}$ \\
Server 4 & $\mathbf{u}+\mathbf{e}_{\nu}$ & $\mathbf{v}$ & $\mathbf{v}$ & $\mathbf{v}$ & $?$
\end{tabular}

Table 3.1. An example of the proposed 1-universal and adaptive robust PIR scheme. The scheme has two stages, with ? indicating the unresponsive server. If server $i$ is unresponsive in stage 1 , the user sends the queries shown in column $i$ of stage 2 to the servers $j \neq i$.

has been studied in the literature in the case of replicated data, and is called robust PIR [2, 7, 13]. We say that a PIR scheme is $r$-robust if it can tolerate $r$ unresponsive or slow servers, the responses of which are replaced with an erasure symbol ?. The erasure symbol ? is absorbing with respect to addition, in the sense that $?+x=$ ? for all $x \in \mathbb{F}_{q}{ }^{2}$. In analogy with the existing work in the literature, we aim at designing $r$-robust PIR schemes that can operate on coded and not only on replicated data. However, we require the additional property that the scheme is universally optimal, in the sense of achieving the maximum PIR rate $R_{P I R}$, i.e., the asymptotic PIR capacity for an $\left[n-r^{\prime}, k\right]$ MDS code, simultaneously for any number of unresponsive servers $r^{\prime}$, up to $r$ of them. Therefore, we avoid having to design the scheme for the worst-case scenario assuming the maximum number of unresponsive servers. We discuss this scheme in detail in Publication II. We will describe the scheme by continuing Example 6 while assuming this additional property.

Example 6 (continued from page 29). Consider the same setting as before. Now, we assume that up to one server might be unresponsive. Therefore, we want to design a universal 1-robust PIR scheme. We propose an adaptive scheme with two stages. The first stage is the same one described in the previous part of this example. If all servers are responsive and fast, the user would stop querying after the first stage. On the other hand, if one of the servers is unresponsive, the user generates and sends a new set of queries to the responsive servers in the second stage. The new queries depend on which server is unresponsive, or deemed slow. In table 3.1, we write explicitly the queries in stage 2 if server $j$ is unresponsive, $j=1, \ldots, 4$.

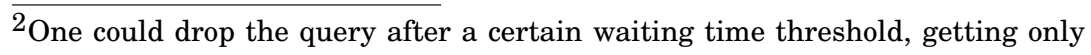
$n-r^{\prime}$ responses for $r^{\prime} \leq r$. 
Next, we describe in detail the case where server 1 is unresponsive in the first stage of the PIR scheme. In this case, the user will not receive the response of server 1, i.e., $\mathbf{u}^{T} A$, which is needed for decoding the desired file in the first stage. Thus, the user will send a second stage of queries to retrieve $\mathbf{u}^{T}$ A and successfully retrieve the desired file symbols. The user can retrieve $\mathbf{u}^{T}$ A from server 3 or server 4 , but if the user asks directly for it in stage 2 , it will reveal $\mathbf{e}_{\nu}$ to the server, thus revealing the identity of the requested file. Hence, the user generates a new random vector $\mathbf{v}=\left[\begin{array}{lll}v_{1} & \ldots & v_{m}\end{array}\right]^{T}$ with elements $\in G F(3)$ to hide the vector $\mathbf{u}$. Implementing the queries in the second column in Table 3.1, the user can decode $\mathbf{u}^{T} A+\mathbf{u}^{T} B$ in stage 2 , and then $f^{\nu}$ using the responses from stage 1 . This scheme achieves the asymptotically optimal PIR rate simultaneously for 0 unresponsive servers $\left(R_{P I R}=1 / 2\right)$ and 1 unresponsive server $\left(R_{P I R}=1 / 3\right)$.

On the other hand, we also construct $t$-private PIR schemes in Publication I when $t \leq n-k$. The PIR scheme uses $n^{\prime}=t+k \leq n$ servers. The user forms the queries by encoding $t$ random vectors using the dual code of the $\left[n^{\prime}, k\right] \operatorname{MDS}$ code $\mathcal{C}$. Recall that for any $\left[n^{\prime}, k\right] \operatorname{MDS}$ code $\mathcal{C}, \mathcal{C} \star \mathcal{C}^{\perp}$ is the $\left[n^{\prime}, n^{\prime}-1\right]$ MDS code. Therefore, the responses live in the $\left[n^{\prime}, n^{\prime}-1\right]$ MDS code, which allows the user to recover one part of the desired file in a single query. To that end, the files will not be subdivided, and a different part will be retrieved in each sub-query. The user sends $k$ sub-queries until the full file is retrieved. Therefore, the PIR rate of this scheme is

$$
R_{P I R}=\frac{1}{k+t} .
$$

This is explained in more detail in the proof of Theorem 2 in Publication I. We illustrate this scheme through Example 7.

Example 7. Consider a DSS using the systematic [5,3] MDS code $\mathcal{C}$ over GF(5) with generator matrix

$$
G=\left[\begin{array}{lllll}
1 & 0 & 0 & 1 & 1 \\
0 & 1 & 0 & 1 & 2 \\
0 & 0 & 1 & 1 & 3
\end{array}\right]
$$

Suppose the system is storing $m=3$ files, such that $f^{i}=\left[\begin{array}{lll}f_{0}^{i} & f_{1}^{i} & f_{2}^{i}\end{array}\right]^{\top}$, $i=1,2,3$. Then, the data is encoded using the matrix $G$ and stored on $n$ servers, as described in Figure 3.4.

The goal in this example is to construct a 2-private PIR scheme on the $D S S$ depicted in Figure 3.4 with $R_{P I R}=\frac{1}{k+t}=\frac{1}{5}$. The scheme consists of $k=3$ sub-queries. 


\begin{tabular}{|c|c|c|c|c|}
\hline Server 1 & Server 2 & Server 3 & Server 4 & Server 5 \\
\hline$f_{0}^{1}$ & $f_{1}^{1}$ & $f_{2}^{1}$ & $f_{0}^{1}+f_{1}^{1}+f_{2}^{1}$ & $f_{0}^{1}+2 f_{1}^{1}+3 f_{2}^{1}$ \\
\hline$f_{0}^{2}$ & $f_{1}^{2}$ & $f_{2}^{2}$ & $f_{0}^{2}+f_{1}^{2}+f_{2}^{2}$ & $f_{0}^{2}+2 f_{1}^{2}+3 f_{2}^{2}$ \\
\hline$f_{0}^{3}$ & $f_{1}^{3}$ & $f_{2}^{3}$ & $f_{0}^{3}+f_{1}^{3}+f_{2}^{3}$ & $f_{0}^{3}+2 f_{1}^{3}+3 f_{2}^{3}$ \\
\hline
\end{tabular}

Figure 3.4. Distributed storage system encoded using the $[5,3]$ MDS code described in Example 7.

Suppose WLOG that the user wants file $f^{1}$. We will explicitly write the first sub-query. Sub-queries 2 and 3 will follow similarly.

The user creates 2 random vectors $\mathbf{u}_{1}$ and $\mathbf{u}_{2} \in \mathbb{F}_{q}^{3}$, and use the dual code $\mathcal{C}^{\perp}$ to encode them and form the random query matrix $D$. The dual code $\mathcal{C}^{\perp}$ has generator matrix

$$
H_{(n-k) \times n}=\left[\begin{array}{ccccc}
-1 & -1 & -1 & 1 & 0 \\
-1 & -2 & -3 & 0 & 1
\end{array}\right] .
$$

According to Definition 2.2.3, the star product $\mathcal{C} \star \mathcal{C}^{\perp}$ for any $M D S$ code $\mathcal{C}$ is the $[n, n-1]$ parity code. Therefore, the response vectors to the random query matrix D are codewords in a [5, 4] MDS code. This allows the user to retrieve $n-k=1$ file symbol by querying the DSS using D. Hence, the user can add a deterministic vector to $D$ in order to retrieve one information symbol. One can see that, following this construction, any two servers will see a random query and they cannot know which file is desired by the user.

This PIR scheme downloads in each sub-query one symbol from each server to recover one information symbol. Therefore, the PIR rate in all 3 sub-queries is $R_{P I R}=\frac{1 \times 3}{5 \times 3}=\frac{1}{5}$.

Moreover, we initiated a line of work in Publication III, where we construct PIR schemes on a DSS with sets of colluding servers, such that some sets of servers might collude, which we call colluding sets. Note that in this case, not any set of $t$ servers, but only certain sets of servers, of possibly different sizes, can collude. This can be motivated by certain servers having geographical proximity, being a local set, user community, etc... In [23], the authors consider a DSS consisting of $m$ files replicated on $n$ servers. The servers are divided into $\zeta$ disjoint colluding sets, where set $j$ consists of $N_{j}$ servers, up to $t_{j}$ of which can collude. The PIR capacity for this setting is:

$$
C_{P I R}=\left(\sum_{i=0}^{m-1}\left(\sum_{j=1}^{\zeta} \frac{t_{j}}{N_{j}}\right)^{-i}\right)^{-1}
$$




\subsection{Regenerating Codes and PIR}

For DSSs where the storage code is a regenerating code, we use the underlying MDS property of PM codes to construct similar schemes as the ones used in Publication I to achieve PIR with a high rate. In Publication $\mathrm{V}$, we construct PIR schemes on such systems, when $t=1$, i.e., the servers do not collude. For this reason, we describe this construction right after the construction of PIR from MDS codes. In [15], the authors construct a multi-file retrieval PIR scheme for product matrix (PM) codes for MBR and MSR codes. In their scheme, $p$ files can be retrieved simultaneously, where $n=p k+d$.

\subsubsection{Minimum Bandwidth Regenerating (MBR) Code Construction}

In Section 2.3.2, we introduced the notion of the MBR extremal point and the product matrix (PM) construction of regenerating codes built to achieve the MBR point. To construct a PIR scheme on a DSS coded using an $(n, k, d)$ regenerating code, we use the symmetric property of PM-MBR codes to achieve a high PIR rate. The scheme in Theorem 1 in Publication $\mathrm{V}$ achieves a PIR rate of

$$
R_{P I R}=\frac{3(n-k)(2 \alpha-k+1)}{6 n \alpha-3 n k+3 n-k^{2}+1} .
$$

As compared to the work in [15], to privately retrieve $p$ files using the scheme in Publication V, the user has to apply the single file PIR scheme $p$ times. We compare the PIR rate in the two schemes for a $(40,7, d) \mathrm{MBR}$ storage code different values of $d$ in Figure 3.5. In the scheme in [15], $n=p k+d$ must hold, thus, $p$ varies with $d$. The larger the number of messages to be downloaded, the larger the PIR rate of [15], but it remains bounded by the scheme in Publication $\mathrm{V}$ for every admissible value of $p$. We also compare the PIR rate achieved by this scheme to the asymptotic PIR capacity of a PIR scheme for an $[n, k]$ MDS coded storage system with no collusion, to the asymptotic capacity of a PIR scheme for an $[n d, B]$ MDS coded storage system with no collusion, and to the conjectured asymptotic capacity of a PIR scheme for an $[n d, B]$ MDS-coded storage system with full $d$-collusion in Figure 3.6. Considering the storage code as an $[n d, B]$ linear code, the PIR protocol must resist to colluding sets of size $\alpha$. Therefore, we can see that one cannot hope to achieve the PIR capacity of an $[n d, B]$ MDS coded storage. 


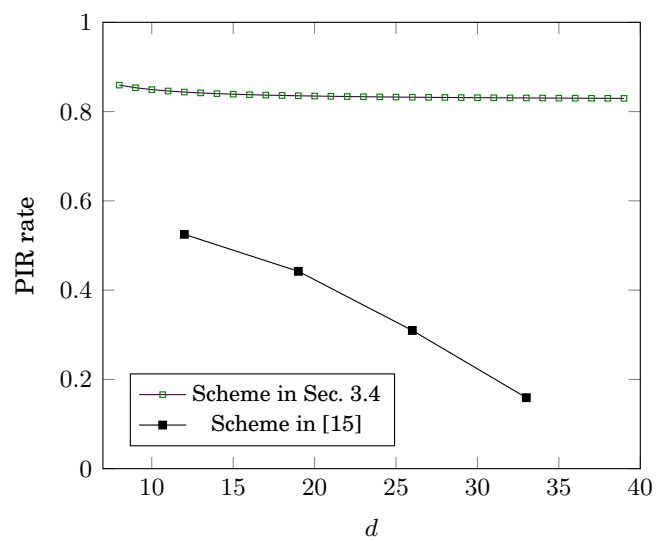

Figure 3.5. PIR rate versus $d$ for the scheme in Publication V and the scheme in [15] with $n=40$ and $k=7$, and $n=p k+d$.

In the scheme we propose for PM-MBR codes in Publication V, we make use of the symmetric property of matrices $\mathbf{M}^{i}$, which allows the user to reuse information in order to decrease the download complexity, i.e., increase the PIR rate. In this work, the servers are assumed not to collude. Each file is subdivided into $L=n-k$ stripes, and $k$ sub-queries are sent to the servers. The $k$ sub-queries are generated in a similar manner to the scheme in Publication I. A sub-query is an $n \times m L$ matrix sent by the user to retrieve information from the servers, such that row $j$ of the query matrix is sent to server $j$. Randomness is embedded in the queries as a way to hide the identity of the user's desired file. Naturally, if privacy were not a concern, a query to retrieve the file $f^{\nu}$ would be the vector of size $n \times m L$ with zeroes everywhere, except in position $\nu$ corresponding to the requested file. We give a simple example to highlight the basic scheme used in this work.

Example 8. We use the $(6,3,4) P M-M B R$ regenerating code described in Example 3. For this purpose, the files are divided into $L=n-k=3$ stripes, and the user sends $k=3$ sub-query vectors:

\begin{tabular}{|l|c|c|c|}
\hline & Sub-query 1 & Sub-query 2 & Sub-query 3 \\
\hline Server 1 & $\mathbf{u}$ & $\mathbf{v}$ & $\mathbf{w}$ \\
\hline Server 2 & $\mathbf{u}$ & $\mathbf{v}$ & $\mathbf{w}$ \\
\hline Server 3 & $\mathbf{u}$ & $\mathbf{v}$ & $\mathbf{w}$ \\
\hline Server 4 & $\mathbf{u}+\mathbf{e}_{3(\nu-1)+1}$ & $\mathbf{v}+\mathbf{e}_{3(\nu-1)+2}$ & $\mathbf{w}+\mathbf{e}_{3(\nu-1)+3}$ \\
\hline Server 5 & $\mathbf{u}+\mathbf{e}_{3(\nu-1)+2}$ & $\mathbf{v}+\mathbf{e}_{3(\nu-1)+3}$ & $\mathbf{w}+\mathbf{e}_{3(\nu-1)+1}$ \\
\hline Server 6 & $\mathbf{u}+\mathbf{e}_{3(\nu-1)+3}$ & $\mathbf{v}+\mathbf{e}_{3(\nu-1)+1}$ & $\mathbf{w}+\mathbf{e}_{3(\nu-1)+2}$ \\
\hline
\end{tabular}

Here, $\mathbf{e}_{(\nu-1) L+s} \in \mathbb{F}_{q}^{m L}$ is the deterministic vector with all zeros, but one 1 
in position $(\nu-1) L+s$, which corresponds to stripe s of what is stored from file $f^{\nu}$. Vectors $\mathbf{u}, \mathbf{v}, \mathbf{w} \in \mathbb{F}_{q}^{m L}$ are vectors picked uniformly at random and sent to each server.

Server responses: All servers project the data stored in rows 3 and 4 on all sub-queries. Then, only servers $2, \ldots, 6$ project the first 2 sub-queries on the data stored in their second row. Lastly, servers $3, \ldots, 6$ project the first sub-query on the data stored in row 1 . Then the servers send their projections back to the user.

We next show that the scheme allows the user to decode the desired file.

- Decodability: For the last $d-k=1$ row, the user receives the projection of all three sub-queries from all six servers. The storage code used to encode the data in the last row is a [6,3] MDS code. The responses to the first subquery from the last row are:

\begin{tabular}{|c|c|}
\hline & Response 1 \\
\hline Server 1 & $\sum_{i=1}^{m} \sum_{s=1}^{3} u_{3(i-1)+s} \cdot\left(f_{s, 7}^{i}+f_{s, 8}^{i}+f_{s, 9}^{i}\right)$ \\
\hline Server 2 & $\sum_{i=1}^{m} \sum_{s=1}^{3} u_{3(i-1)+s} \cdot\left(f_{s, 7}^{i}+2 f_{s, 8}^{i}+4 f_{s, 9}^{i}\right)$ \\
\hline Server 3 & $\sum_{i=1}^{m} \sum_{s=1}^{3} u_{3(i-1)+s} \cdot\left(f_{s, 7}^{i}+3 f_{s, 8}^{i}+2 f_{s, 9}^{i}\right)$ \\
\hline Server 4 & $\sum_{i=1}^{m} \sum_{s=1}^{3} u_{3(i-1)+s} \cdot\left(f_{s, 7}^{i}+4 f_{s, 8}^{i}+2 f_{s, 9}^{i}\right)+f_{1,7}^{\nu}+4 f_{1,8}^{\nu}+2 f_{1,9}^{\nu}$ \\
\hline Server 5 & $\sum_{i=1}^{m} \sum_{s=1}^{3} u_{3(i-1)+s} \cdot\left(f_{s, 7}^{i}+5 f_{s, 8}^{i}+4 f_{s, 9}^{i}\right)+f_{2,7}^{\nu}+5 f_{2,8}^{\nu}+4 f_{2,9}^{\nu}$ \\
\hline Server6 & $\sum_{i=1}^{m} \sum_{s=1}^{3} u_{3(i-1)+s} \cdot\left(f_{s, 7}^{i}+6 f_{s, 8}^{i}+f_{s, 9}^{i}\right)+f_{3,7}^{\nu}+6 f_{3,8}^{\nu}+f_{3,9}^{\nu}$ \\
\hline
\end{tabular}

From the above table, we can see that the user can recover the three random symbols

$$
\begin{aligned}
& \sum_{i=1}^{m} \sum_{s=1}^{3} u_{3(i-1)+s} \cdot f_{s, 7}^{i} \\
& \sum_{i=1}^{m} \sum_{s=1}^{3} u_{3(i-1)+s} \cdot f_{s, 8}^{i}
\end{aligned}
$$

and

$$
\sum_{i=1}^{m} \sum_{s=1}^{3} u_{3(i-1)+s} \cdot f_{s, 9}^{i}
$$

along with the three sums of information symbols

$$
\begin{gathered}
f_{1,7}^{\nu}+4 f_{1,8}^{\nu}+2 f_{1,9}^{\nu}, \\
f_{2,7}^{\nu}+5 f_{2,8}^{\nu}+4 f_{2,9}^{\nu}, \\
f_{3,7}^{\nu}+6 f_{3,8}^{\nu}+f_{3,9}^{\nu} .
\end{gathered}
$$

Following the same reasoning, from the second and third sub-queries the user can retrieve the random symbols

$$
\sum_{i=1}^{m} \sum_{s=1}^{3} v_{3(i-1)+s} f_{s, 7}^{i}, \sum_{i=1}^{m} \sum_{s=1}^{3} w_{3(i-1)+s} f_{s, 7}^{i}
$$




$$
\sum_{i=1}^{m} \sum_{s=1}^{3} v_{3(i-1)+s} \cdot f_{s, 8}^{i}, \sum_{i=1}^{m} \sum_{s=1}^{3} w_{3(i-1)+s} \cdot f_{s, 8}^{i}
$$

and

$$
\sum_{i=1}^{m} \sum_{s=1}^{3} v_{3(i-1)+s} \cdot f_{s, 9}^{i}, \sum_{i=1}^{m} \sum_{s=1}^{3} w_{3(i-1)+s} \cdot f_{s, 9}^{i},
$$

along with the sums of required symbols,

$$
\begin{array}{cc}
f_{2,7}^{\nu}+4 f_{2,8}^{\nu}+2 f_{2,9}^{\nu}, & f_{3,7}^{\nu}+4 f_{3,8}^{\nu}+2 f_{3,9}^{\nu}, \\
f_{3,7}^{\nu}+5 f_{3,8}^{\nu}+4 f_{3,9}^{\nu}, & f_{1,7}^{\nu}+5 f_{1,8}^{\nu}+4 f_{1,9}^{\nu}, \\
f_{1,7}^{\nu}+6 f_{1,8}^{\nu}+f_{1,9}^{\nu}, & f_{2,7}^{\nu}+6 f_{2,8}^{\nu}+f_{2,9}^{\nu} .
\end{array}
$$

From the information sums, the user can retrieve the desired symbols $f_{7}^{\nu}, f_{8}^{\nu}$, and $f_{9}^{\nu}$. Notice that the PIR scheme run over the fourth column achieves a PIR rate of $3 / 6$.

For the third row, the storage code is a [6,4] MDS code. Recall that the user has already collected information in the responses from row 4. As a consequence, the user knows the vector $f_{9}^{\nu}$ as well as the random symbols

$$
\begin{gathered}
\sum_{i=1}^{m} \sum_{s=1}^{3} u_{3(i-1)+s} \cdot f_{s, 9}^{i}, \\
\sum_{i=1}^{m} \sum_{s=1}^{3} v_{3(i-1)+s} \cdot f_{s, 9}^{i},
\end{gathered}
$$

and

$$
\sum_{i=1}^{m} \sum_{s=1}^{3} w_{3(i-1)+s} \cdot f_{s, 9}^{i}, .
$$

Therefore, the responses from the third row allow the user to decode the symbols, just like from the responses from the last row. The user, thus, recovers $f_{3}^{\nu}, f_{5}^{\nu}$, and $f_{6}^{\nu}$ with a rate $3 / 6$.

For the second row, the storage code is also a [6,4] MDS code, but the user can use the information she collected from rows 3 and 4. Thus, the user does not need the response from server 1 in order to decode the symbols. It means that the code can be assumed to be reduced to a $[5,2] M D S$ code. The user can then decode the parts $f_{2}^{\nu}$, and $f_{4}^{\nu}$ from servers $2, \ldots, 6$ and from the first 2 sub-queries, with rate $6 / 10=3 / 5$.

Following the same reasoning for the first row, the user needs the responses of servers $3, \ldots, 6$ to the first sub-query only. The storage code can be seen as a $[4,1]$ repetition code on those servers, after introducing the already known information. This allows the user to decode the last part of the file, $f_{1}^{\nu}$, with rate $3 / 4$. 


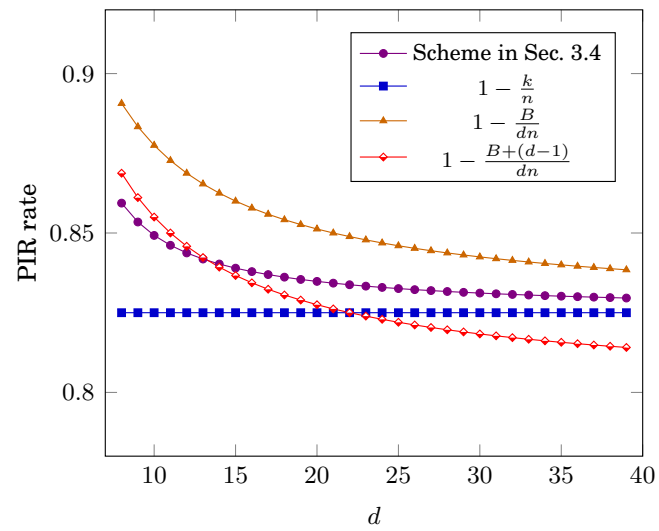

Figure 3.6. PIR rate versus $d$ when $n=40$. Comparison between the PIR rate of the scheme in the current work, the capacity $1-k / n$ of a PIR scheme for an $[n, k]$ MDS coded storage system with no collusion, the capacity $1-B / n d$ of a PIR scheme for an $[n d, B]$ MDS coded storage system with no collusion, and the conjectured capacity $1-(B+d-1) / n d$ of a PIR scheme for an $[n d, B]$ MDS-coded storage system with full $d$ collusion.

Finally, the PIR rate of the scheme in this example is $R_{\mathrm{MBR}}=\frac{3+6+9+9}{4+10+18+18}=$ $\frac{27}{50}=0.54$. We see that this rate is larger than $1-\frac{k}{n}=1-\frac{3}{6}=\frac{1}{2}=0.5$ which is the capacity of scalar MDS-coded PIR schemes, but less than $1-\frac{B}{n d}=1-\frac{9}{6 \times 4}=\frac{5}{8}=0.625$, which is an upper bound on the capacity of $[n d, B]$-coded PIR schemes.

- Privacy: Privacy follows from the fact that every server sees a uniform random vector.

\subsubsection{Minimum Storage Regenerating (MSR) Code Construction}

In Section 2.3.3, we introduced the notion of the MSR extremal point and the construction to achieve this minimum storage point. For the MSR point, we construct a PIR scheme in Publication V, where we consider $d=2 k-2$ for simplicity. The PIR scheme achieves a PIR rate

$$
R_{P I R}=\frac{3(n-2 \alpha)}{3 n-2 \alpha+2}
$$

which is between $1-d / n$, the rate obtained by Dorkson and $\mathrm{Ng}$ [15] which is also the PIR capacity of an $[n, d]$ MDS code, and $1-k / n$, the PIR capacity of an $[n, k]$ MDS code. We compare the rates for $n=40$ and $d=2 k-2$ in Figure 3.7.

Example 9. We use the $(6,3,4) P M-M S R$ regenerating code presented in Example 4, with $\alpha=2$. Files are divided into $L=n-2 \alpha=2$ stripes, and 


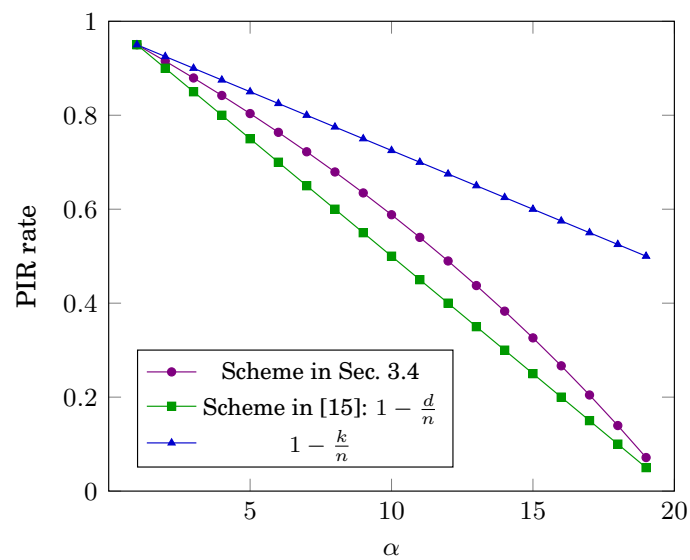

Figure 3.7. PIR rate versus $\alpha$ in the MSR case, for fixed $n=40$ with $2 \alpha=d=2 k-2$.

the user sends $2 \alpha=4$ sub-queries:

\begin{tabular}{|l|c|c|c|c|}
\hline & Sub-query 1 & Sub-query 2 & Sub-query 3 & Sub-query 4 \\
\hline Server 1 & $\mathbf{u}$ & $\mathbf{v}$ & $\mathbf{w}$ & $\mathbf{y}$ \\
\hline Server 2 & $\mathbf{u}$ & $\mathbf{v}$ & $\mathbf{w}$ & $\mathbf{y}$ \\
\hline Server 3 & $\mathbf{u}$ & $\mathbf{v}$ & $\mathbf{w}+\mathbf{e}_{(\nu-1) L+1}$ & $\mathbf{y}+\mathbf{e}_{(\nu-1) L+2}$ \\
\hline Server 4 & $\mathbf{u}$ & $\mathbf{v}$ & $\mathbf{w}+\mathbf{e}_{(\nu-1) L+2}$ & $\mathbf{y}+\mathbf{e}_{(\nu-1) L+1}$ \\
\hline Server 5 & $\mathbf{u}+\mathbf{e}_{(\nu-1) L+1}$ & $\mathbf{v}+\mathbf{e}_{(\nu-1) L+2}$ & $\mathbf{w}$ & $\mathbf{y}$ \\
\hline Server 6 & $\mathbf{u}+\mathbf{e}_{(\nu-1) L+2}$ & $\mathbf{v}+\mathbf{e}_{(\nu-1) L+1}$ & $\mathbf{w}$ & $\mathbf{y}$ \\
\hline
\end{tabular}

The vector $\mathbf{e}_{(\nu-1) L+s} \in \mathbb{F}_{q}^{F \times(n-2 \alpha)}$ is the all zero vector with a single 1 in position $(\nu-1) L+s$, i.e., indicating stripe sfrom file $f^{\nu}$. Vectors $\mathbf{u}, \mathbf{v}, \mathbf{w}, \mathbf{y} \in$ $\mathbb{F}_{q}^{m L}$ are random uniformly distributed vectors.

The servers project the data stored in row 2 on all the queries. Servers 1 and 2 do not respond to any other queries. Servers $3, \ldots, 6$ project only the first 2 sub-queries on the data stored in the first row.

\subsection{PIR with Byzantine Servers}

In addition to constructing PIR schemes on a DSS with replicated data, assuming up to $t$ colluding and $r$ unresponsive servers, much work has been done assuming that some servers are Byzantine (malicious) or unsynchronized, and thus return erroneous information to the user $[2,7,13$, 17]. In [3], the PIR capacity for a DSS consisting of $m$ files replicated on $n$ servers and up to $t$ colluding and $b$ byzantine servers was found to be

$$
C_{P I R}=\frac{n-2 b}{n} \cdot \frac{1-\frac{t}{n-2 b}}{1-\left(\frac{t}{n-2 b}\right)^{m}} .
$$




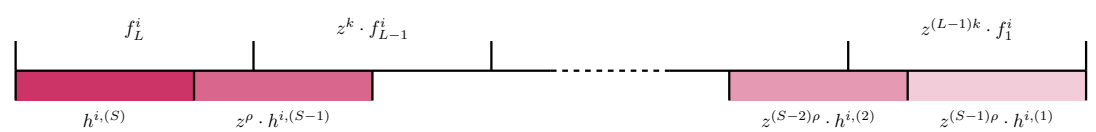

Figure 3.8. Partitioning the polynomial $f_{\ell}^{i}$ into $S$ blocks of size $\rho$ (bottom) and $L$ blocks of size $k$ each (top).

For coded storage, the $t$-private PIR schemes on MDS coded data with $n$ servers constructed in Publication I use only $n^{\prime}=k+t$ of the servers. In [18], the authors construct $t$-private PIR schemes using RS codes on $n$ servers, using the star product between RS codes, as discussed in Definition 2.2.3. Using some techniques from [18], we construct a scheme for PIR on RS coded data that allows for $b$ byzantine servers, $r$ unresponsive servers, and $t$ colluding servers in Publication IV.

The DSS encodes $m$ files using an $[n, k]$ RS storage code $\mathcal{C}$, as described in Section 2.2.2. We will briefly describe the PIR scheme, then give a simple example to highlight its basic properties. We will assume that the byzantine servers respond with an arbitrary element in $\mathbb{F}_{q}^{\ell}$, and the unresponsive servers that respond with an erasure symbol ?. In this work, we assume that after $n-r$ servers respond, the user cuts the other $r$ slower servers.

As in previous schemes, the files are subdivided into $L$ stripes, and $S$ sub-queries are sent to the servers to retrieve a complete file. In every sub-query $s$, we choose the query codewords from the RS code $\mathcal{D}=R S[n, t]$. To correct $b$ errors and $r$ erasures, the response vector should live in a code with minimum distance $d_{\text {min }}=2 b+r+1$. Recall from Definition 2.2.3 that the star product of any two RS codes $\mathcal{C}$ and $\mathcal{D}$ with the same evaluation points is an RS code, i.e., $\mathcal{C} \star \mathcal{D}=R S[n, k+t-1]$. Thus, among the $n$ dimensions of the response vector, $k+t-1$ dimensions are spanned by the randomness. Moreover, $2 b+r$ dimensions are required for error correction, and therefore, $\rho=n-(k+t-1)-(2 b+r)$ dimensions are left for information retrieval.

For divisibility purposes, the files are subdivided into

$$
L=\frac{\operatorname{LCM}(\rho, k)}{k}
$$

stripes and the user sends

$$
S=\frac{\operatorname{LCM}(\rho, k)}{\rho}
$$

sub-queries. Thus, $L k=S \rho$, which allows the polynomial $f_{\ell}^{i}$ to be partitioned in two different ways, as shown in Figure 3.8. 


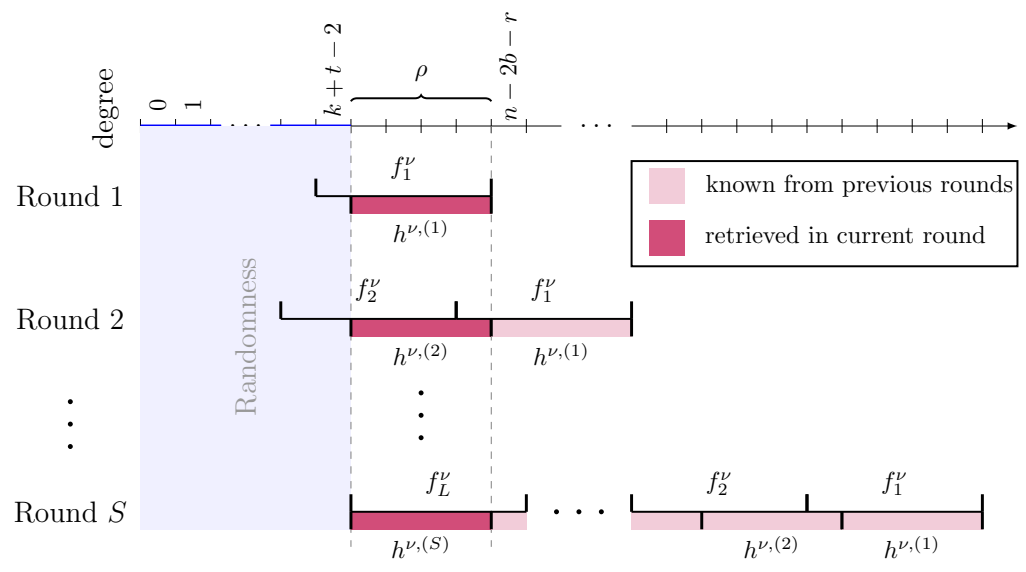

Figure 3.9. General Scheme for retrieving file $f^{\nu}$.

$$
\begin{aligned}
& f^{\nu} \\
& \begin{array}{|c|c|}
\overbrace{f_{1,0}^{\nu}+f_{1,1}^{\nu} z}+f_{1,2}^{\nu} z^{2}+f_{1,3}^{\nu} z^{3} \\
\hline f_{1,0}^{\nu}+f_{1,1}^{\nu} z & z^{2}\left(f_{1,2}^{\nu}+f_{1,3}^{\nu} z\right)
\end{array} \\
& h^{\nu,(2)} \quad z^{2} \cdot h^{\nu,(1)}
\end{aligned}
$$

Figure 3.10. Divisions for Example 10. The file $f^{\nu}$ is split into two parts $h^{\nu,(1)}$ and $h^{\nu,(2)}$, which are retrieved in rounds 1 and 2 , respectively.

Assuming the user would like to retrieve file $f^{\nu}$ privately, she then adds the evaluation of another polynomial to the query, such that all $\rho$ coefficients of a polynomial $h^{\nu,(s)}(z)$ are all retrieved in round $s$. A schematic of the scheme is shown in Figure 3.9, and the scheme is explained in more detail in Section III.B in Publication IV. The scheme achieves a PIR rate

$$
R_{P I R}=\frac{n-k-t-2 b-r-1}{n-r}
$$

which we conjecture is the asymptotic PIR capacity in this setting.

We next give a simple example to highlight the scheme basics.

Example 10. Suppose a DSS with parameters $n=9, k=4, t=b=$ $r=1$. Let the storage code $\mathcal{C}$ be an $[9,4]$ Reed-Solomon code. Let $\alpha$ be the evaluation vector of the Reed-Solomon code. To correct the errors and erasures, the responses should live in a code with a minimum distance $d_{\min } \geq 2 b+r+1=4$, allowing it to tolerate b errors and $r$ erasures. Among the $n=9$ total dimensions of a single response, $k+t-1=4$ dimensions are spanned by randomness required to preserve privacy and introduced by the storage code, and $2 b+r$ dimensions are required to perform erasure 


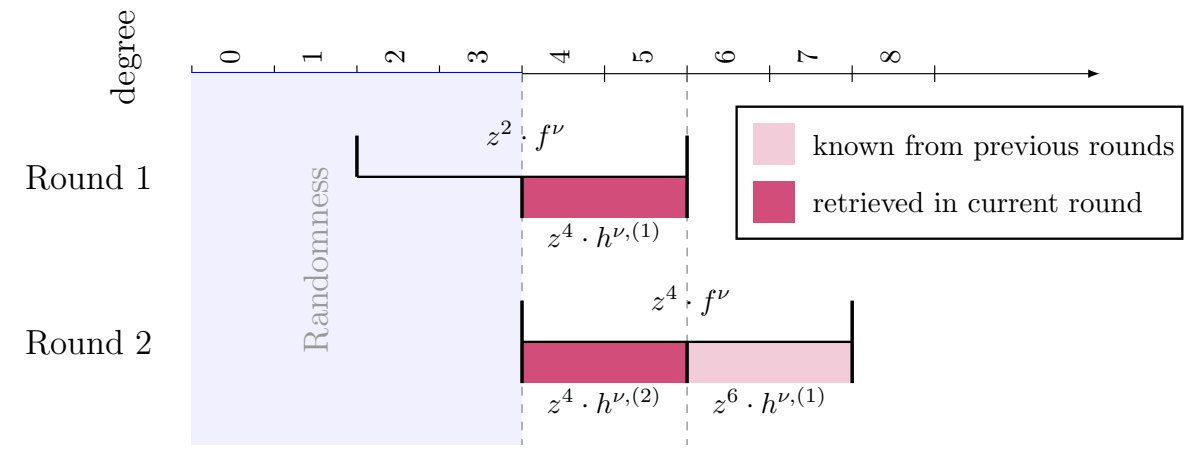

Figure 3.11. Retrieval scheme construction for Example 10. The file $f^{\nu}$, consisting of 4 coefficients, is retrieved over 2 rounds.

and error correction. Hence, no more than the remaining $\rho:=n-(k+t+$ $2 b+r-1)=2$ dimensions can be used to retrieve information. According to (3.8) and (3.9), the files will be subdivided into $L=1$ stripe (thus we drop the subscript $\ell$ ) and the scheme will require $S=2$ rounds to retrieve $S \rho=4$ symbols.

Suppose that the user wishes to download file $f^{\nu}$ privately. Based on the above discussion, in each round the user can recover exactly $\rho=2$ symbols of file $f^{\nu}$. We see that in this example, since file $f^{\nu}$ has one stripe, we can write

$$
\begin{aligned}
f^{\nu}(z) & =f_{1,0}^{\nu}+f_{1,1}^{\nu} z+f_{1,2}^{\nu} z^{2}+f_{1,3}^{\nu} z^{3} \\
& =h^{\nu,(2)}(z)+h^{\nu,(1)}(z) z^{2}
\end{aligned}
$$

where $h^{\nu,(1)}(z)=f_{1,2}^{\nu}+f_{1,3}^{\nu} z$ and $h^{\nu,(2)}(z)=f_{1,0}^{\nu}+f_{1,1}^{\nu} z$, as depicted in Figure 3.10. In round s of the scheme, the user can retrieve the coefficients of $h^{\nu,(s)}(z)$, which comprise $\rho$ symbols of the desired file, see Figure 3.11.

In round 1, a random coefficient (constant polynomial) $d^{i,(1)}$ is chosen for every file $f^{i}, 1 \leq i \leq m$. For the requested file, $f^{\nu}$, the monomial $z^{2}$ will be added to $d^{\nu,(1)}$. In other words, we define

$q^{(1)}(z)=\left(q^{1,(1)}(z), \ldots, q^{m,(1)}(z)\right), \quad$ where $\quad q^{i,(1)}(z)=\left\{\begin{array}{ll}d^{i,(1)} & \text { if } i \neq \nu \\ d^{i,(1)}+z^{2} & \text { for } i=\nu\end{array}\right.$.

Then, the query $q_{j}$ sent to the $j^{\text {th }}$ server is given by

$$
q_{j}^{(1)}=q^{(1)}\left(\alpha_{j}\right)=\left(q^{1,(1)}\left(\alpha_{j}\right), \ldots, q^{m,(1)}\left(\alpha_{j}\right)\right)=\left\{\begin{array}{ll}
d^{i,(1)} & \text { if } i \neq \nu \\
d^{i,(1)}+\alpha_{j}^{2} & \text { if } i=\nu
\end{array} .\right.
$$

Without the errors and erasures induced by the Byzantine and unrespon- 
sive servers, the response from server $j$ is

$$
\begin{aligned}
r_{j}^{(1)}=\left\langle q_{j}^{(1)}, y_{j}\right\rangle & =\sum_{i=1}^{m} q^{i,(1)}\left(\alpha_{j}\right) f^{i}\left(\alpha_{j}\right) \\
& =\sum_{i=1}^{m} d^{i,(1)} f^{i}\left(\alpha_{j}\right)+\alpha_{j}^{2} f^{\nu}\left(\alpha_{j}\right) .
\end{aligned}
$$

The vector $r^{(1)}=\left(r_{1}^{(1)}, \ldots, r_{n}^{(1)}\right)$ consisting of all of the responses from the $n$ servers is therefore

$$
r^{(1)}=\operatorname{eval}\left(r^{(1)}(z)\right), \quad \text { where } \quad r^{(1)}(z)=\sum_{i=1}^{m} d^{i,(1)} f^{i}(z)+z^{2} f^{\nu}(z) .
$$

Since $\operatorname{deg}\left(r^{(1)}(z)\right) \leq 5$, we see that $r^{(1)}$ is an element of a Reed-Solomon code with parameters $[9,6]$ with minimum distance 4 , and can thus tolerate the one error and one erasure introduced by the Byzantine and unresponsive servers, respectively.

Expanding the polynomial $r^{(1)}(z)$, we have

$$
\begin{aligned}
r^{(1)}(z) & =\sum_{i=1}^{m} d^{i,(1)} f^{i}(z)+z^{2} f^{\nu}(z) \\
& =\underbrace{\sum_{i=1}^{m} d^{i,(1)} f^{i}(z)+h^{\nu,(2)}(z) z^{2}}_{=: g(z), \operatorname{deg} \leq 3}+h^{\nu,(1)}(z) z^{4} .
\end{aligned}
$$

Since $\operatorname{deg}(g(z)) \leq 3$, we have that $\operatorname{eval}(g(z)) \in \mathcal{C}$. Therefore, we can recover the coefficients of $h^{\nu, 1}(z)$, namely $f_{1,2}^{\nu}$ and $f_{1,3}^{\nu}$, from the response vector $r^{(1)}$.

In round 2 , we again sample $m$ constant polynomials $d^{i,(2)}$ and set

$q^{(2)}(z)=\left(q^{1,(2)}(z), \ldots, q^{m,(2)}(z)\right), \quad$ where $\quad q^{i,(2)}(z)= \begin{cases}d^{i,(2)} & \text { if } i \neq \nu \\ d^{i,(2)}+z^{4} & \text { if } i=\nu .\end{cases}$

A similar calculation as in the first round shows that the total response vector $r^{(2)}$ in the second round, before the addition of errors and erasures, is $r^{(2)}=\operatorname{eval}\left(r^{(2)}(z)\right)$, where

$$
r^{(2)}(z)=\underbrace{\sum_{i=1}^{m} d^{i,(1)} f^{i}(z)}_{\operatorname{deg} \leq 3}+h^{\nu,(2)}(z) z^{4}+\underbrace{h^{\nu,(1)}(z) z^{6}}_{\text {known from round } 1} .
$$

The user receives eval $\left(r^{(2)}(z)\right)$ plus a vector consisting of errors and erasures. From round 1, the user knows $h^{\nu,(1)}(z)$, and before correcting for errors and erasures can subtract $\operatorname{eval}\left(h^{\nu,(1)}(z) z^{6}\right)$ from what they receive. What is left is a codeword of a Reed-Solomon code, which can correct the errors and erasures as in round 1. Again as in round 1, the user reads off the coefficients of $h^{\nu,(2)}(z)$, namely $f_{1,0}^{\nu}$ and $f_{1,1}^{\nu}$. 
The user now has all of the coefficients of $f^{\nu}$ and can reconstruct the entire file. The rate of this scheme is clearly seen to be

$$
\frac{n-r-(k+t+2 b-1)}{n-r}=\frac{\rho}{n-r}=\frac{2}{8}=\frac{1}{4} .
$$

\subsubsection{Symmetric PIR scheme}

A PIR scheme is symmetric if the user, while retrieving the requested file $f^{\nu}$, gains no information about any of the other files $f^{i}$ for $i \neq \nu$. Symmetric PIR was studied in [42], which deals with the case of $b=r=0$, as well as in the scheme of [43], which deals with the case $k=1$.

We extend the above scheme to a symmetric variant by assuming the servers have access to a joint source of randomness. Each round, the joint source of randomness outputs a uniform random codeword $\pi^{(s)}=$ $\operatorname{eval}\left(\pi^{(s)}(z)\right)$, where $\pi^{(s)}(z) \in \mathbb{F}[z]^{<k+t-1}$.

The symmetric variant in Publication IV uses the minimum amount of shared randomness and achieves the same rate as the asymmetric PIR variant, which we also conjecture is the capacity of a symmetric PIR scheme on MDS coded data in this setting.

The symmetric PIR scheme follows exactly as before, with the distinction that all servers compute the responses back to the user by adding a common "secret" $\pi^{(s)}$. The responses of the non-Byzantine servers would then be $r_{j}^{(s)}=\left\langle q_{j}^{(s)}, y_{j}\right\rangle+\pi_{j}^{(s)}$. As before, the user receives an erasure symbol from the unresponsive servers, and an arbitrary element of $\mathbb{F}_{q}$ from the Byzantine servers. Since $\operatorname{deg}\left(\pi^{(s)}(z)\right)<k+t-1$, it is absorbed into the 'randomness' term $g^{(s)}(z)$ and therefore does not affect how the user recovers the $\rho$ information symbols. Since $\pi^{(s)}(z)$ is uniformly chosen, there is clearly no information leaked about any files $f^{i}$ for $i \neq \nu$.

\subsection{Network Coding with PIR}

The use of networks as a communication channel between the user and the servers made way to considering a scheme that allows the user to retrieve a file from a set of servers through a network privately. In Publication VI, we assume a random linear network over which a user communicates with $l$ servers storing data using a Gabidulin code $\mathcal{G}(n, k)$. We assume a random linear network as defined in Section 2.4.

As shown in Figure 2.2, we assume a DSS with $l$ servers, storing $m$ files $f^{1}, \ldots, f^{m} \in \mathbb{F}_{q}^{s \times k}$, where each file $f^{i}$ is subdivided into $L$ stripes. The data 
$\mathbf{f} \in \mathbb{F}_{q^{s}}^{m L \times k}$ is defined as follows

$$
\mathbf{f}=\Phi^{-1}\left(\begin{array}{c}
f^{1} \\
f^{2} \\
\vdots \\
f^{m}
\end{array}\right) \in \mathbb{F}_{q^{s}}^{m L \times k}
$$

where $\Phi^{-1}$ denotes the one-to-one mapping from $s \times k$ matrices in $\mathbb{F}_{q}$ to vectors in $\mathbb{F}_{q^{s}}^{k}$ (i.e., the inverse mapping of $\Phi$ ). Every row of $\mathbf{f} \in \mathbb{F}_{q^{s}}^{m L \times k}$ is encoded with a $\mathcal{G}(n, k)$ code over $\mathbb{F}_{q^{s}}$ to obtain $m L$ Gabidulin codewords, contained in the rows of $Y \in \mathbb{F}_{q^{s}}^{m L \times n}$.

Assume that the data is stored on $l$ servers where $n$ is divisible by $l$. We divide the matrix $Y$ into $l$ blocks of $\rho \triangleq n / l$ columns and store each such block on a separate server. We denote by $Y_{j} \in \mathbb{F}_{q^{s}}^{m L \times \rho}$ the $j^{\text {th }}$ block of $Y$, stored on server $j$. The servers are assumed to be honest-but-curious, and at most $t$ of them may collude, i.e., share the queries that they receive with each other.

According to the system model, each server is storing $\rho$ columns of $Y$, which means the privacy constraint should take into account that each server acts as $\rho$ colluding "sub-servers". We can view the system as a set of $n$ sub-servers, such that column $j$ of $Y$ is stored on sub-server $j$, and up to $\rho t$ servers may collude. Technically, the collusion in this setting is not full $\rho t$-collusion as not any $\rho t$ sub-servers can collude, but we assume full $\rho t$ collusion for simplicity.

In this work, we first assume an error-free network, where only erasures may occur on the random network matrix, and then move on to a network where both errors and erasures may occur.

Assume that $s$ divides $m$, and that the user wants file $f^{\nu}$. The files are subdivided into $L=n-k-\rho t+1$ stripes, and a file will be retrieved in $k$ rounds of queries. In sub-query round $i$, the user generates $t \rho$ random vectors of length $m L$, denoted by $u_{1}^{(i)}, \ldots, u_{t \rho}^{(i)} \in \mathbb{F}_{q}^{m L}$, such that

$$
U^{(i)}=\left(\begin{array}{c}
u_{1}^{(i)} \\
\vdots \\
u_{t \rho}^{(i)}
\end{array}\right)^{\top}
$$

where $U^{(i)} \in \mathbb{F}_{q}^{m L \times t \rho}$. These vectors are then mapped onto $t \rho$ vectors of size $\mu=m L / s$ in $\mathbb{F}_{q^{s}}$, forming matrix $U^{(i) \prime}$. The vectors are chosen uniformly at random so that the queries received by any $t \rho$ servers reveal no information about the index of the requested file. 

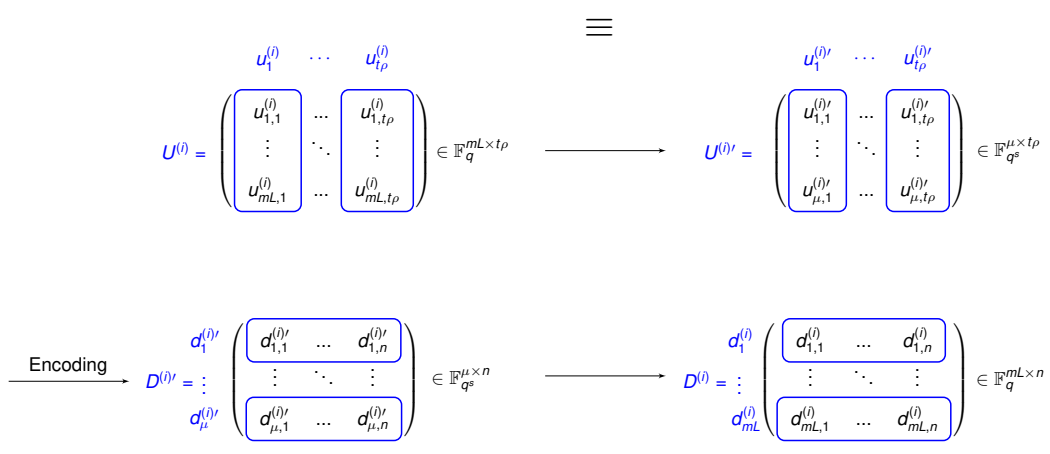

Figure 3.12. Encoding random vectors into a query matrix.

The user encodes the $\mu$ rows of $U^{(i) \prime}$ into $\mu$ codewords of the $\mathcal{G}(n, t \rho)$ Gabidulin code $\mathcal{D}$ to generate the matrix $D^{(i) \prime} \in \mathbb{F}_{q^{s}}^{\mu \times n}$. Those $\mu$ codewords are then mapped onto $m L$ codewords of $\mathcal{D}$ to form the matrix $D^{(i)} \in \mathbb{F}_{q}^{m L \times n}$, as shown in Fig. 3.12.

We briefly describe the PIR scheme for an error-free network in Section 3.6.1 and for a network with errors in Section 3.6.2.

\subsubsection{Error-Free Network}

Assuming the network introduces no errors in the system, the queries will follow in the same manner as in an RS code. The difference is that the query codewords are now chosen from a Gabidulin code $\mathcal{G}(n, \rho t)$, and the servers respond by using the Gabidulin star product between the query code and the storage code. We assume that some erasures might occur. The probability that the responses are received by the user with no erasures is the probability that the random network matrices have full rank. We denote the overall random channel transfer matrix in the network from the user to the servers in sub-query $i$ by $A^{(i)} \in \mathbb{F}_{q^{s}}^{n \times n}$ and the overall random channel transfer matrix from the servers to the user in round $i$ by $A^{(i) \prime} \in \mathbb{F}_{q^{s}}^{n \times n}$.

Based on Proposition 2, the probability that both matrices $A^{(i)}$ and $A^{(i) \prime}$ have full rank in all query rounds $i=1, \ldots, k$ is $\left(1-\frac{1}{q^{s}}\right)^{2 n+k}$. Since the scheme is the same for all rounds, we will drop the superscript $(i)$ in the following. We prove the following theorem in Publication VI (Thm 1).

Theorem 1. In the above setting, the user can decode the file $f^{\nu}$ from the $k$ responses from rounds $i=1, \ldots, k, R_{\text {reo }}^{\nu}$ with PIR rate

$$
R_{P I R}=\left(1-\frac{1}{q^{s}}\right)^{2 n+k}\left(1-\frac{k+t \rho-1}{n}\right) .
$$

We then quantify the probability of an erasure and give the PIR rate 
assuming some erasures occur on the network in Theorem 2 in Publication VI.

We give here a simple example.

Example 11. Let $m$ denote the number of files. The data, $\mathbf{f} \in \mathbb{F}_{q^{s}}^{m \times 2}$, is encoded using a $\mathcal{G}(3,2)$ Gabidulin code $\mathcal{C}$ over $\mathbb{F}_{2^{3}}$, where

$$
\mathcal{C}=\left\{\left(f(1), f(\alpha), f\left(\alpha^{2}\right)\right): f(z)=f_{0} z+f_{1} z^{2}\right\}
$$

Hence, its corresponding generator matrix is

$$
G_{\mathcal{C}}=\left(\begin{array}{ccc}
1 & \alpha & \alpha^{2} \\
1 & \alpha^{2} & \alpha^{2}+\alpha
\end{array}\right) \in \mathbb{F}_{q^{s}}^{2 \times 3}
$$

We consider a network with $l=3$ servers, where every column $Y_{j}$ of

$$
Y=X G_{\mathcal{C}} \in \mathbb{F}_{q^{s}}^{m \times 3}
$$

is stored on a server.

Let us construct a PIR scheme where the user wants file $f^{\nu}$ from $\mathbf{f}$, while keeping the identity $\nu$ of the file hidden from the servers, and the servers do not collude, i.e., $t=1$. The number of subdivisions in this case is $L=$ $n-k-t \rho+1=1$. WLOG, assume the user wants file $f^{1}$. To this end, we use a $\mathcal{G}(3,1)$ code as the query code,

$$
\mathcal{D}=\left\{\left(f(1), f(\alpha), f\left(\alpha^{2}\right)\right): f(z)=f_{0} z\right\}
$$

whose generator matrix is

$$
G_{D}=\left(\begin{array}{ccc}
1 & \alpha & \alpha^{2}
\end{array}\right) \in \mathbb{F}_{q^{s}}^{1 \times 3} .
$$

Two rounds are needed to retrieve the full file $f^{1}$, hence, let matrix $e_{1}=$ $\left(\begin{array}{llll}1 & 0 & \cdots & 0\end{array}\right)$. Then, $E^{1,(1)}$ is the $3 \times m$ matrix $E^{1,(1)}=\left(\begin{array}{c}e_{1} \\ \mathbf{0}_{n-1 \times m}\end{array}\right)$, and $E^{1,(2)}=\left(\begin{array}{c}\mathbf{0}_{1 \times m} \\ e_{1} \\ \mathbf{0}_{n-2 \times m}\end{array}\right)$ which the user uses to decode the file $f^{1}$. The matrix $E^{1,(i)}$ is added to the random matrix $D^{(i)}$, which is chosen uniformly at random from the code $\mathcal{D}$.

The query matrix is then lifted,

$$
Q_{j}^{1,(i)}=\left(\begin{array}{l}
1 \\
i
\end{array} D_{Q_{j}}^{1,(i)}\right)
$$

where $D_{Q_{j}}^{1,(i)}$ is the $j^{\text {th }}$ row of $D_{Q}^{1,(i)}$, generated in round $i$, for $i=1,2$. 
In round $i$, the user sends row $j$ of matrix $Q^{f,(i)}$ to server $j$. Assume that server $j$ receives

$$
Q_{j, \text { rec }}^{f,(i)}=\left(\begin{array}{l:l}
a_{j}^{(i)} & a_{j}^{(i)} D_{Q_{j}}^{1,(i)}
\end{array}\right),
$$

where $a_{j}^{(i)} \in \mathbb{F}_{2^{3}}$ is a scalar introduced by the network in round $i$.

There are two cases a server can encounter:

- $a_{j}^{(i)}=0$ : In this case, server $j$ receives the all-zero vector and sends nothing back to the user.

- $a_{j}^{(i)} \neq 0$ : In this case, server $j$ will calculate the star product of its contents $Y_{j}$ with the received query matrix, $Q_{j, r e c}^{f,(i)}$, and lifts the obtained matrix to form its response

$$
R_{j}^{1,(i)}=\left(\begin{array}{l:l:l}
1 & a_{j}^{(i)} & a_{j}^{(i)} D_{Q_{j}}^{1,(i)}
\end{array}\right),
$$

which is sent to the user. The user receives

$$
R_{j, r e c}^{1,(i)}=\left(\begin{array}{l:l:}
a_{j}{ }^{(i) \prime}: a_{j}{ }^{(i) \prime} a_{j}^{(i)}: a_{j}{ }^{(i) \prime} a_{j}^{(i)} D_{Q_{j}}^{1,(i)}
\end{array}\right)
$$

from server $j, j=1,2,3$, and forms the overall matrix

$$
R^{1,(i)}=\left(\begin{array}{l:l:l}
A^{(i) \prime} & A^{(i) \prime} A^{(i)} & A^{(i) \prime}\left(Y \star A^{(i)} D_{Q_{1}}^{1,(i)}\right)
\end{array}\right)
$$

where $A^{(i) \prime}=\left(\begin{array}{ccc}a_{1}^{(i) \prime} & 0 & 0 \\ 0 & a_{2}^{(i) \prime} & 0 \\ 0 & 0 & a_{3}^{(i) \prime}\end{array}\right)$ and $A^{(i)}=\left(\begin{array}{ccc}a_{1}^{(i)} & 0 & 0 \\ 0 & a_{2}^{(i)} & 0 \\ 0 & 0 & a_{3}^{(i)}\end{array}\right)$.

The user may now face two different scenarios:

- All coefficients $a_{j}^{(i) \prime} \neq 0$ for $j \in\{1,2,3\}$. In this case, the user can decode the matrix

$$
\left(Y \star D_{Q_{1}}^{1,(i)}\right)=\left(Y \star D^{(i) \top}+Y \star E^{1,(i)}\right) .
$$

The matrix $Y \star D^{(i) \top}$ is a codeword from a $\mathcal{G}(3,2)$ code $\mathcal{C} \star \mathcal{D}$, since $Y$ is a codeword from a $\mathcal{G}(3,2)$ code and $D^{(i)}$ is from a $\mathcal{G}(3,1)$ code. Therefore, the user can decode one rank erasure in $\mathcal{C} \star \mathcal{D}$. In our setting, the matrix $E_{j}^{1,(i)}$ is the deterministic matrix introduced by the user in order to decode the desired file. The positions of the errors are known, so they can be viewed as erasures, thus allowing the user to decode $Y \star E^{1,(i)}$.

- On the other hand, if at least one of the coefficients $a_{j}{ }^{(i) \prime}=0$, then $A^{(i) \prime}\left(Y \star D^{(i) \top}\right)$ becomes a codeword in a $\mathcal{G}(2,2)$ Gabidulin code that is not able to correct any errors or erasures. Thus, the user 
will not be able to decode the desired file. This case is equivalent to the case where $a_{j}{ }^{(i)}=0$. For instance, assuming $a_{2}^{\prime(i)}=0$, the user obtains $R^{1,(i)}=\left(\begin{array}{l:l:l}A^{(i) \prime} & A^{(i) \prime} A^{(i)} & A^{(i) \prime}\left(Y \star A^{(i)} D_{Q_{1}}^{1,(i)}\right)\end{array}\right)$, such that the second row is the all-zero vector, and one can observe that the user cannot decode the file from this response matrix.

In this example, when the decoding is successful, the PIR rate is $\frac{1}{3}$. Otherwise, the PIR rate is zero. The probability that none of the $a_{j}{ }^{(i)}$ 's and the $a_{j}^{(i) '}$ 's are zero in both rounds, and thus, that decoding is successful, is $\left(\left(1-\frac{1}{q^{s}}\right)^{6}\right)^{2}=\left(1-\frac{1}{8}\right)^{12}$. Therefore, the PIR rate of this scheme is $\frac{1}{3}\left(1-\frac{1}{q^{s}}\right)^{12}=\frac{1}{3}\left(\frac{7}{8}\right)^{12} \approx 0.067$. We see here that the PIR rate approaches the asymptotically optimal PIR rate $\frac{1}{3}$ when $q$ is sufficiently large.

\subsubsection{Network with Errors}

Errors in a random network happen, e.g., due to malicious servers injecting erroneous packets or due to congestion. In this section, we will consider such an erroneous network. The network is assumed to introduce up to $\epsilon$ errors and up to $\tau$ erasures to the sent codewords.

From [37], it is known that errors of rank $\epsilon$ can be corrected if $2 \epsilon \leq$ $\tau+d_{\text {min }}-1$, where $d_{\text {min }}$ is the minimum rank distance of the code. For this purpose, each file is subdivided into $L=n-k-\rho t-2 \epsilon-\tau+1$ stripes. The matrix $D^{(i)}$ is constructed in the same manner as before. As for the matrix $E^{\nu,(i)}$, it is chosen as a codeword of a rank metric code $\mathcal{E}^{\nu}$ such that the response $\mathcal{C} \star\left(\mathcal{D}+\mathcal{E}^{\nu}\right)$ is again a Gabidulin code, which follows from the techniques used in Publication IV. Specifically, we start with $E^{\nu}$ being the $n \times m L$ all-zero matrix. In round $i$, for all $\delta \leq\left\lceil\frac{i L}{k}\right\rceil$, the vector $e_{\delta}^{\nu,(i)}$ is taken to be the vector with all zeros and a single 1 at position $L(\nu-1)+\delta$, which is the vector requesting the stripe $\delta$ of the file $f^{\nu}$. The vector $e_{\delta}^{\nu,(i)}$ is encoded using a $\mathcal{G}(n, 1)$ code $\mathcal{E}^{\nu}=\left\{g\left(\alpha_{0}\right), \cdots, g\left(\alpha_{n-1}\right): g(z)=g_{0} z^{q^{i L-\delta k+k+\rho t-1}}\right\}$ and added to row $(\nu-1) m L+\delta$ of $E^{\nu,(i)} 3$.

Remark 1. In the above scheme, the storage code $\mathcal{C}$ is a Gabidulin code of dimension $k$. Hence, we pick $\mathcal{D}$ to be a Gabidulin code of dimension $t \rho$, so that $\mathcal{C} \star \mathcal{D}$ is a Gabidulin code of dimension $t \rho+k-1$, as discussed in Definition 2.5.1. Thus, in round $i, \mathcal{C} \star\left(\mathcal{D}+\mathcal{E}^{\nu}\right)$ is a $\mathcal{G}(n, k+t \rho+L-1)$ Gabidulin code, padded with parts retrieved from previous rounds $1, \ldots, i-1$, with degrees $>n-1$, where degrees $q^{t \rho+k}$ and higher of the evaluation polynomial consist of parts of the file requested by the user. From $\mathcal{C} \star\left(\mathcal{D}+\mathcal{E}^{\nu}\right)$, the

\footnotetext{
${ }^{3}$ For more specific details on how the scheme is constructed, see Publication IV.
} 
user can interpolate to retrieve the higher powers, thus retrieving $L$ parts of the file she requires.

We now assume that $2 \epsilon+\tau \leq d_{\text {min }}-1$, where $d_{\text {min }}$ is the minimum distance of the code $\mathcal{C} \star\left(\mathcal{D}+\mathcal{E}^{\nu}\right)$. Then $\epsilon$ errors and $\tau$ erasures can be corrected. In this case, the underlying PIR scheme would be similar to the scheme in Publication IV, with the ability to correct $\epsilon$ errors and $\tau$ erasures. We assume that $2 \epsilon+\tau \leq d_{\text {min }}-1$. For the PIR scheme for a network assuming errors and erasures may occur, the query code is constructed assuming a certain number of errors and erasures introduced by the network, thus, in this section, the probability of error is not taken into account. 


\section{Conclusions}

The main focus of this thesis is providing privacy to users retrieving information from coded storage systems. Given the current technological advancements, larger amounts of data need to be stored reliably for long periods of time. With that, the use of coded storage becomes essential. Coded distributed storage systems provide reliability and availability of data without increasing the storage overhead to the extent of replicating the data on multiple servers.

With the advantages of using distributed storage systems comes a concern for user privacy. Private information retrieval (PIR) allows a user to retrieve a file or multiple files from a distributed storage system without revealing the identities of the files to any of the servers. On the other hand, symmetric PIR allows the user to privately retrieve a file from the storage system without getting any extra information about the undesired files.

In this thesis, we studied the problem of constructing PIR schemes on various distributed storage systems. We focused on optimizing the PIR rate as the number of files in the system is asymptotically large. The constructed PIR scheme on MDS coded data achieves the asymptotic PIR capacity given in [4]. Also, for a storage system using an RS code, allowing for colluding, byzantine, and unresponsive servers, we constructed a scheme which achieves the asymptotic PIR capacity for all the cases where the capacity is known. Moreover, this scheme was extended to a symmetric PIR scheme. For regenerating codes, we built a PIR scheme with a higher PIR rate than the known rate for the same system. Finally, we studied the problem of retrieving a file privately from a distributed storage system over a random linear network. In this setting, we constructed a PIR scheme achieving a high PIR rate.

The work on PIR is far from complete and many research questions are 
still open. One of the most intriguing problems is quantifying the PIR capacity for coded storage systems in the presence of colluding, byzantine, and unresponsive servers. 


\section{Bibliography}

[1] R. Ahlswede, N. Cai, S.-Y. R. Li, and R. W. Yeung. Network Information Flow. IEEE Transactions on Information Theory, 46(4):1204-1216, 2000.

[2] Daniel Augot, Françoise Levy-Dit-Vehel, and Abdullatif Shikfa. A StorageEfficient and Robust Private Information Retrieval Scheme Allowing Few Servers. In Cryptology and Network Security, pages 222-239. Springer, 2014.

[3] Karim Banawan and Sennur Ulukus. The Capacity of Private Information Retrieval from Byzantine and Colluding Databases. IEEE Transactions on Information Theory, 2018.

[4] Karim Banawan and Sennur Ulukus. The Capacity of Private Information Retrieval from Coded Databases. IEEE Transactions on Information Theory, 64(3):1945-1956, 2018.

[5] Amos Beimel and Yuval Ishai. Information-Theoretic Private Information Retrieval: A Unified Construction. In Automata, Languages and Programming, pages 912-926. Springer, 2001.

[6] Amos Beimel, Yuval Ishai, Eyal Kushilevitz, and Jean-François Raymond. Breaking the $O\left(n^{1 /(2 k-1)}\right)$ Barrier for Information-Theoretic Private Information Retrieval. In The 43rd Annual IEEE Symposium on Foundations of Computer Science, 2002. Proceedings., pages 261-270. IEEE, 2002.

[7] Amos Beimel and Yoav Stahl. Robust Information-Theoretic Private Information Retrieval. In Security in Communication Networks, pages 326-341. Springer, 2003.

[8] Christian Cachin, Silvio Micali, and Markus Stadler. Computationally Private Information Retrieval with Polylogarithmic Communication. In International Conference on the Theory and Applications of Cryptographic Techniques, pages 402-414. Springer, 1999.

[9] T.H. Chan, Siu-Wai Ho, and H. Yamamoto. Private Information Retrieval for Coded Storage. In 2015 IEEE International Symposium on Information Theory (ISIT), pages 2842-2846. IEEE, June 2015.

[10] B Chor, O Goldreich, E. Kushilevitz, and M Sudan. Private Information Retrieval. In IEEE Symposium on Foundations of Computer Science, pages 41-50, 1995. 
[11] T. M. Cover and J. A. Thomas. Elements of Information Theory. WileyInterscience, 2006.

[12] P. Delsarte. Bilinear Forms over a Finite Field with Applications to Coding Theory. J. Combinatorial Theory Ser. A, 25(3):226-241, 1978.

[13] Casey Devet, Ian Goldberg, and Nadia Heninger. Optimally Robust Private Information Retrieval. In USENIX Security Symposium, pages 269-283, 2012.

[14] A. Dimakis, P. Godfrey, Y. Wu, M. Wainright, and K. Ramchandran. Network Coding for Distributed Storage Systems. IEEE Transactions on Information Theory, 56(9):4539-4551, Sep. 2010.

[15] Chatdanai Dorkson and Siaw-Lynn Ng. Multi-Message Private Information Retrieval using Product-Matrix MSR and MBR Codes. CoRR, abs/1808.02023, 2018.

[16] Zeev Dvir and Sivakanth Gopi. 2 Server PIR with Sub-Polynomial Communication. In Proceedings of the Forty-Seventh Annual ACM on Symposium on Theory of Computing, STOC '15, pages 577-584, New York, NY, USA, 2015. ACM.

[17] G. Fanti and K. Ramchandran. Multi-Server Private Information Retrieval over Unsynchronized Databases. In Communication, Control, and Computing (Allerton), 2014 52nd Annual Allerton Conference on, pages 437-444, Sept 2014.

[18] Ragnar Freij-Hollanti, Oliver W. Gnilke, Camilla Hollanti, and David A. Karpuk. Private Information Retrieval from Coded Databases with Colluding Servers. SIAM J. Appl. Algebra Geometry, 1(1):647-664, 2017.

[19] Ernest Mukhamedovich Gabidulin. Theory of Codes with Maximum Rank Distance. Problemy Peredachi Informatsii, 21(1):3-16, 1985.

[20] Greenberg, Andy. The Grand TOR: How To Go Anonymous Online.

[21] T. Ho, M. Medard, R. Koetter, D. R. Karger, M. Effros, J. Shi, and B. Leong. A Random Linear Network Coding Approach to Multicast. IEEE Transactions on Information Theory, 52(10):4413-4430, Oct 2006.

[22] Yuval Ishai, Eyal Kushilevitz, Rafail Ostrovsky, and Amit Sahai. Batch Codes and their Applications. In Proceedings of the thirty-sixth annual ACM symposium on Theory of computing, pages 262-271. ACM, 2004.

[23] Zhuqing Jia, Hua Sun, and Syed A Jafar. The Capacity of Private Information Retrieval with Disjoint Colluding Sets. In GLOBECOM 2017-2017 IEEE Global Communications Conference, pages 1-6. IEEE, 2017.

[24] Ralf Koetter and Frank R Kschischang. Coding for Errors and Erasures in Random Network Coding. IEEE Transactions on Information Theory, 54(8):3579-3591, 2008.

[25] Siddhartha Kumar, Hsuan-Yin Lin, Eirik Rosnes, and Alexandre Graell I Amat. Achieving Private Information Retrieval Capacity in Distributed Storage using an Arbitrary Linear Code. arXiv preprint arXiv:1712.03898, 2017. 
[26] Eyal Kushilevitz and Rafail Ostrovsky. Replication is not Needed: Single Database, Computationally-Private Information Retrieval. In FOCS, page 364. IEEE, 1997.

[27] Eyal Kushilevitz and Rafail Ostrovsky. One-way trapdoor permutations are sufficient for non-trivial single-server private information retrieval. In International Conference on the Theory and Applications of Cryptographic Techniques, pages 104-121. Springer, 2000.

[28] Hsuan-Yin Lin, Siddhartha Kumar, Eirik Rosnes, et al. On the fundamental limit of private information retrieval for coded distributed storage. arXiv preprint arXiv:1808.09018, 2018.

[29] Florence Jessie MacWilliams and Neil James Alexander Sloane. The Theory of Error-Correcting Codes. Elsevier, 1977.

[30] Umberto Martínez-Peñas and Ruud Pellikaan. Rank Error-Correcting Pairs. Designs, Codes and Cryptography, 84(1-2):261-281, 2017.

[31] M. Medard, M. Effros, T. Ho, and D. R. Karger. On Coding For NonMulticast Networks. In Proceedings of 41st Annual Allerton Conference on Communication, Control, and Computing, October 2003.

[32] Andreas Pfitzmann and Marit Köhntopp. Anonymity, Unobservability, and Pseudonymity - A Proposal for Terminology. In Designing privacy enhancing technologies, pages 1-9. Springer, 2001.

[33] Korlakai Vinayak Rashmi, Nihar B Shah, and P Vijay Kumar. Optimal Exact-Regenerating Codes for Distributed Storage at the MSR and MBR Points via a Product-Matrix Construction. IEEE Transactions on Information Theory, 57(8):5227-5239, 2011.

[34] Irving S Reed and Gustave Solomon. Polynomial Codes over Certain Finite Fields. Journal of the society for industrial and applied mathematics, 8(2):300-304, 1960.

[35] R. M. Roth. Maximum-Rank Array Codes and their Application to Crisscross Error Correction. IEEE Trans. Inform. Theory, 37(2):328-336, March 1991.

[36] NB Shah, KV Rashmi, and Kannan Ramchandran. One Extra Bit of Download Ensures Perfectly Private Information Retrieval. In 2014 IEEE International Symposium on Information Theory, pages 856-860. IEEE, 2014.

[37] Danilo Silva, Frank R Kschischang, and Ralf Koetter. A Rank-Metric Approach to Error Control in Random Network Coding. IEEE transactions on information theory, 54(9):3951-3967, 2008.

[38] Hua Sun and Syed Ali Jafar. The Capacity of Private Information Retrieval. IEEE Transactions on Information Theory, 63(7):4075-4088, 2017.

[39] Hua Sun and Syed Ali Jafar. The Capacity of Robust Private Information Retrieval With Colluding Databases. IEEE Trans. Information Theory, 64(4):2361-2370, 2018.

[40] Hua Sun and Syed Ali Jafar. The Capacity of Symmetric Private Information Retrieval. IEEE Transactions on Information Theory, 2018. 
[41] Tor Project: Anonymity Online. https://www.torproject.org/.

[42] Qiwen Wang and Mikael Skoglund. Linear Symmetric Private Information Retrieval for MDS Coded Distributed Storage with Colluding Servers. In 2017 IEEE Information Theory Workshop (ITW), pages 71-75. IEEE, 2017.

[43] Qiwen Wang and Mikael Skoglund. Secure Symmetric Private Information Retrieval from Colluding Databases with Adversaries. In Communication, Control, and Computing (Allerton), 2017 55th Annual Allerton Conference on, pages 1083-1090. IEEE, 2017.

[44] Qiwen Wang and Mikael Skoglund. Symmetric Private Information Retrieval for MDS Coded Distributed Storage. In 2017 IEEE International Conference on Communications (ICC), pages 1-6. IEEE, 2017.

[45] S. Yekhanin. Private Information Retrieval. Magazine Communications of the ACM, 2010. 


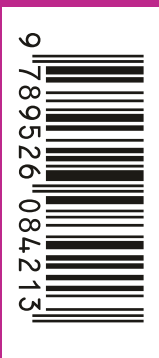

ISBN 978-952-60-8421-3 (printed)

ISBN 978-952-60-8422-0 (pdf)

ISSN 1799-4934 (printed)

ISSN 1799-4942 (pdf)

Aalto University

School of Science

Department of Mathematics and Systems Analysis

www.aalto.fi

\author{
BUSINESS + \\ ECONOMY \\ ART + \\ DESIGN + \\ ARCHITECTURE \\ SCIENCE + \\ TECHNOLOGY \\ CROSSOVER
}

DOCTORAL

DISSERTATIONS 